\title{
On the Passivity and Positivity Properties in Dynamic Systems: Their Achievement under Control Laws and Their Maintenance under Parameterizations Switching
}

\author{
M. De la Sen (iD \\ Institute of Research and Development of Processes (IIDP), University of the Basque Country, Campus of Leioa, \\ P.O. Box 48940, Leioa, Bizkaia, Spain \\ Correspondence should be addressed to M. De la Sen; manuel.delasen@ehu.eus
}

Received 4 August 2017; Accepted 28 November 2017; Published 24 January 2018

Academic Editor: Viliam Makis

Copyright (C) 2018 M. De la Sen. This is an open access article distributed under the Creative Commons Attribution License, which permits unrestricted use, distribution, and reproduction in any medium, provided the original work is properly cited.

\begin{abstract}
This paper is devoted to discuss certain aspects of passivity results in dynamic systems and the characterization of the regenerative systems counterparts. In particular, the various concepts of passivity as standard passivity, strict input passivity, strict output passivity, and very strict passivity (i.e., joint strict input and output passivity) are given and related to the existence of a storage function and a dissipation function. Later on, the obtained results are related to external positivity of systems and positivity or strict positivity of the transfer matrices and transfer functions in the time-invariant case. On the other hand, how to achieve or how eventually to increase the passivity effects via linear feedback by the synthesis of the appropriate feed-forward or feedback controllers or, simply, by adding a positive parallel direct input-output matrix interconnection gain is discussed.
\end{abstract}

\section{Introduction}

This paper is devoted to discuss certain aspects of passivity results in dynamic systems and the characterization of the regenerative versus passive systems counterparts. In particular, the various concepts of passivity as standard passivity, strict input passivity, strict output passivity, and very strict passivity (i.e., joint strict input and output passivity) are given and related to the existence of a storage function and a dissipation function. Basic previous background concepts on passivity are given in [1-4] and some related references therein. More detailed generic results about passivity and positivity are given in [5-7]. Note, in particular, the use of passive devices is very relevant in certain physical and electronic applications. See, for instance, [8]. Later on, the obtained results are related to external positivity of systems and positivity or strict positivity of the transfer matrices and transfer functions in the time-invariant case. On the other hand, it is discussed and formalized how to proceed in the case of passivity failing. It is also analyzed the way of eventually increasing the passivity effects via linear feedback by the synthesis of the appropriate feed-forward or feedback controllers or, simply, by adding a positive parallel direct input-output matrix interconnection gain having a minimum positive lower-bounding threshold gain which is also a useful idea for asymptotic hyperstability of parallel disposals of systems, [9]. For the performed analysis, the concept of relative passivity index, which is applicable for both passive and nonpassive systems, is addressed and modified to a lower number by the use of appropriate feedback or feed-forward compensators. Finally, the concept of passivity is discussed for switched systems which can have both passive and nonpassive configurations which become active governed by switching functions. The passivity property is guaranteed by the switching law under a minimum residence time at passive active configurations provided that the first active configuration of the switched disposal is active and that there are no two consecutive active nonpassive configurations in operation. Some illustrative examples are also discussed. The so-called storage functions which play a relevant role in the study of passivity are Lyapunov functions. Lyapunov functions are commonly used in the background literature for stability analysis of deterministic and dynamic systems. See, for instance, [10-12]. 


\subsection{Notation}

(i) $\mathbf{R}_{0+}=\mathbf{R}_{+} \cup\{0\}$, where $\mathbf{R}_{+}=\{r \in \mathbf{R}: r>0\}, \bar{p}=$ $\{1,2, \ldots, p\}$,

(ii) $D>0$ denotes that the real matrix $D$ is positive definite while $D \geq 0$ denotes that it is positive semidefinite,

(iii) $\lambda_{\min }(\cdot)$ and $\lambda_{\max }(\cdot)$ denote, respectively, the minimum and maximum eigenvalues of the real symmetric $(\cdot)$ matrix,

(iv) $\widehat{G} \in\{\mathrm{PR}\}$ denotes that the transfer matrix $\widehat{G}(s)$ of a linear time-invariant system is positive real; that is, $\widehat{G}(s)+\widehat{G}^{T}(-s) \geq 0$ for all $\operatorname{Re} s>0$, and $\widehat{G} \in\{\mathrm{SPR}\}$ denotes that it is strictly positive real; that is, $\widehat{G}(s)+$ $\widehat{G}^{T}(-s)>0$ for all Re $s \geq 0$. The set of strongly positive real transfer matrices $\{\mathrm{SSPR}\}$ is the subset of $\{\mathrm{SPR}\}$ of entries having relative degree zero so that they cannot diverge as $|s| \rightarrow \infty$. If the linear time -invariant system is a SISO one (i.e., it has one input and one output) then $\widehat{G} \in\{\mathrm{PR}\}$ if $\operatorname{Re} \widehat{G}(s) \geq 0$ for all $\operatorname{Re} s>0$ and $\widehat{G} \in\{\mathrm{SPR}\}$ if $\operatorname{Re} \widehat{G}(s)>0$ for all $\operatorname{Re} s \geq 0$,

(v) A dynamic system is positive (resp., externally positive) if all the state components (resp., if all the output components) are nonnegative for all time $t \geq 0$ for any given nonnegative initial conditions and nonnegative input,

(vi) $\mathbf{i}=\sqrt{-1}$ is the complex unity,

(vii) $I_{m}$ is the $m$ th identity matrix,

(viii) The superscript $T$ stands for matrix transposition,

(ix) $\mathbf{H}_{\infty}$ is the Hardy space of all complex-valued functions $F(s)$ of a complex variable $s$ which are analytic and bounded in the open right half-plane $\operatorname{Re} s>0$ of $\operatorname{norm}\|F\|_{\infty}=\sup \{|F(s)|: \operatorname{Re} s>0\}=\sup \{|F(\mathbf{i} \omega)|:$ $\omega \in \mathbf{R}\}$ (by the maximum modulus theorem) and $\mathbf{R} \mathbf{H}_{\infty}$ is the subset of real-rational functions of $\mathbf{H}_{\infty}$.

\section{Preliminaries}

Consider a dynamic system $G: \mathrm{H}_{e} \rightarrow \mathrm{H}_{e}$ with state $x \in \mathbf{R}^{n}$, input $u \in \mathbf{R}^{m}$, and output $y \in \mathbf{R}^{m}$, where $\mathrm{H}_{e}$ is the extended space of the Hilbert space $\mathrm{H}$ endowed with the inner product $\langle\cdot, \cdot\rangle$ from $\mathrm{H}_{e} \times \mathrm{H}_{e}$ to $\mathbf{R}$ consisting of the truncated functions $u_{t}(\tau)=u(\tau)$ for $\tau \in[0, t]$ and $u_{t}(\tau)=0 ; \forall t, \tau(>t) \in \mathbf{R}_{0+}$ and $u: \mathbf{R}_{0+} \rightarrow \mathbf{R}^{m}$. If $u \in \mathrm{H}_{e}$ then

$$
\begin{aligned}
\left\|u_{t}\right\|_{2}^{2} & =\langle u, u\rangle_{t}=\left\langle u_{t}, u_{t}\right\rangle=\int_{0}^{t} u^{T}(\tau) u(\tau) d \tau \\
& =\int_{0}^{\infty} u_{t}^{T}(\tau) u_{t}(\tau) d \tau ; \quad \forall t \geq 0 .
\end{aligned}
$$

Definition 1 (see [2]). The above dynamic system is

(1) $L_{2}$-stable if $u \in L_{2}^{m}$ implies $G u \in L_{2}^{m}$;
(2) nonexpansive if $\exists \lambda$ and $\exists \gamma>0$ s.t. for all $u \in \mathrm{H}_{e}$,

$\int_{0}^{t}(G u)^{T}(\tau) u(\tau) d \tau \leq \lambda+\gamma^{2} \int_{0}^{t} u^{T}(\tau) u(\tau) d \tau ;$

$\forall t \geq 0$;

0 ;

(3) passive if $\exists \varepsilon \geq 0$ such that $\int_{0}^{t} y^{T}(\tau) u(\tau) d \tau \geq-\varepsilon ; \forall t \geq$

(4) strictly input passive if $\exists \varepsilon \geq 0$ and $\exists \varepsilon_{u}>0$ s.t.

$\int_{0}^{t} y^{T}(\tau) u(\tau) d \tau \geq-\varepsilon+\varepsilon_{u} \int_{0}^{t} y^{T}(\tau) u(\tau) d \tau ;$

$\forall t \geq 0$;

(5) strictly output passive if $\exists \beta \geq 0$ and $\exists \varepsilon_{y}>0$ s.t.

$\int_{0}^{t} y^{T}(\tau) u(\tau) d \tau \geq-\varepsilon+\varepsilon_{y} \int_{0}^{t} y^{T}(\tau) y(\tau) d \tau ;$

$\forall t \geq 0$;

(6) strictly input/output passive (or very strictly passive) if $\exists \beta \geq 0, \exists \varepsilon_{u}>0$ and $\exists \varepsilon_{y}>0$ s.t.

$$
\begin{aligned}
\int_{0}^{t} y^{T}(\tau) u(\tau) d \tau \geq & -\varepsilon+\varepsilon_{u} \int_{0}^{t} y^{T}(\tau) u(\tau) d \tau \\
& +\varepsilon_{y} \int_{0}^{t} y^{T}(\tau) y(\tau) d \tau ; \quad \forall t \geq 0 .
\end{aligned}
$$

The constants $\varepsilon, \varepsilon_{u}$, and $\varepsilon_{y}$ are, respectively, referred to as the passivity, input passivity, and output passivity constants.

\section{Some Passivity and Positivity Results: Passivity Achievement by Direct Input-Output Interconnection}

Note that the above definitions can be expressed equivalently via an inner product notation. Note also that the above definitions are equivalent for $\varepsilon=0$ to the corresponding positivity and strict positivity concepts [1] as mentioned in [2]. In particular, some relevant positivity and passivity properties are summarized in the following result for a singleinput single-output (SISO) system by relating the time and frequency domains descriptions:

Theorem 2. Consider a linear time-invariant SISO (i.e., $m=$ 1) system whose transfer function $\widehat{G} \in\{P R\}$. Then, the following properties hold:

(i) $\int_{0}^{t} y(\tau) u(\tau) d \tau \geq 0$ and $y(t) u(t) \geq 0 ; \forall t \geq 0$ and, furthermore, if $u \in L_{2}$ then $y \in L_{2}$. Then, the system is passive.

(ii) Assume, in addition, that $\widehat{G} \in\{S P R\}$. Then $\gamma \geq$ $\int_{0}^{t} y(\tau) u(\tau) d \tau \geq \varepsilon_{u} \int_{0}^{t}|u(\tau)|^{2} d \tau-\varepsilon$ for any $t \in(0, \infty]$ and some $\gamma, \varepsilon \in \mathbf{R}_{0+}$.

(iii) If, furthermore, the system is externally positive then $\gamma \geq \int_{0}^{t} y(\tau) u(\tau) d \tau>0 ; \forall t \geq 0$ for any given nonnegative initial conditions and nonnegative input. 
(iv) Define $R_{\widehat{G}}=\|(1-\widehat{G}(\mathbf{i} \omega)) /(1+\widehat{G}(\mathbf{i} \omega))\|_{\infty}=$ $\sup _{\omega \in \mathbf{R}_{0+}}|(1-\widehat{G}(\mathbf{i} \omega)) /(1+\widehat{G}(\mathbf{i} \omega))|$ as the relative passivity index of the transfer function $\widehat{G}(s)=\widehat{N}(s) / \widehat{D}(s) \in \mathbf{R H}_{\infty}(\widehat{N}(s)$ and $\widehat{D}(s)$ being the numerator and denominator polynomials of $\widehat{G}(s))$. Then, the constraint $a_{G} \leq R_{\widehat{G}}=\|\left(\widehat{D}_{G}(\mathbf{i} \omega)-\widehat{N}_{G}(\mathbf{i} \omega)\right) /$ $\left(\widehat{D}_{G}(\mathbf{i} \omega)+\widehat{N}_{G}(\mathbf{i} \omega)\right) \|_{\infty} \leq b_{G}$ is guaranteed for some $a_{G}, b_{G}(\geq$ $\left.a_{G}\right) \in \mathbf{R}_{0+}$ if

$$
\begin{aligned}
& \frac{1-a_{G}^{2}}{2\left(1+a_{G}^{2}\right)}\left(\operatorname{Re}^{2} \widehat{D}_{G}(\mathbf{i} \omega)+\operatorname{Re}^{2} \widehat{N}_{G}(\mathbf{i} \omega)\right) \\
& \quad \geq \operatorname{Re} \widehat{D}_{G}(\mathbf{i} \omega) \operatorname{Re} \widehat{N}_{G}(\mathbf{i} \omega)+\operatorname{Im} \widehat{D}_{G}(\mathbf{i} \omega) \operatorname{Im} \widehat{N}_{G}(\mathbf{i} \omega) \\
& \quad \geq \frac{1-b_{G}^{2}}{2\left(1+b_{G}^{2}\right)}\left(\operatorname{Re}^{2} \widehat{D}_{G}(\mathbf{i} \omega)+\operatorname{Re}^{2} \widehat{N}_{G}(\mathbf{i} \omega)\right)
\end{aligned}
$$

$\forall \omega \in \mathbf{R}_{0+}$.

If $b_{G} \leq 1$ (resp., $b_{G}<1$ ) then $\widehat{G} \in\{P R\}$ (resp., $\widehat{G} \in\{S P R\}$ ).

Proof. It turns out that the Fourier transforms (denoted with hats and the same symbols as their time functions counterparts) of the truncated input and output for any time exist since the truncated signals are in $L_{2}$. Therefore, Parseval's theorem can be applied to express $\int_{0}^{t} y(\tau) u(\tau) d \tau \geq 0 ; \forall t \geq$ 0 in the frequency domain. Take into account, in addition, that the hodograph of the frequency system's response $\widehat{G}(\mathbf{i} \omega)$ satisfies $\operatorname{Re} \widehat{G}(\mathbf{i} \omega)=\operatorname{Re} \widehat{G}(-\mathbf{i} \omega)$ and $\operatorname{Im} \widehat{G}(\mathbf{i} \omega)=-\operatorname{Im} \widehat{G}(-\mathbf{i} \omega)$ for all $\omega \in(-\infty, \infty)$ and that $\min _{\omega \in \mathbf{R}_{0+}} \operatorname{Re} \widehat{G}(\mathbf{i} \omega) \geq 0$ since $\widehat{G} \in\{P R\}$. Thus, the various expressions which follow hold under zero initial conditions of the dynamic system:

$$
\begin{aligned}
& \int_{0}^{t} y(\tau) u(\tau) d \tau=\int_{0}^{\infty} y_{t}(\tau) u_{t}(\tau) d \tau \\
& =\frac{1}{2 \pi} \int_{-\infty}^{\infty} \widehat{y}_{t}(\mathbf{i} \omega) \widehat{u}_{t}(-\mathbf{i} \omega) d \omega \\
& =\frac{1}{2 \pi} \int_{-\infty}^{\infty} \widehat{G}(\mathbf{i} \omega)\left|\widehat{u}_{t}(\mathbf{i} \omega)\right|^{2} d \omega \\
& =\frac{1}{2 \pi} \min _{\omega \in \mathbf{R}_{0+}} \operatorname{Re} \widehat{G}(\mathbf{i} \omega) \int_{-\infty}^{\infty}\left|\widehat{u}_{t}(\mathbf{i} \omega)\right|^{2} d \omega \\
& \geq \min _{\omega \in \mathbf{R}_{0+}} \widehat{G}(\mathbf{i} \omega) \int_{-\infty}^{\infty}\left|\widehat{u}_{t}(\tau)\right|^{2} d \tau \\
& =\min _{\omega \in \mathbf{R}_{0+}} \operatorname{Re} \widehat{G}(\mathbf{i} \omega) \int_{-\infty}^{\infty}\left|u_{t}(\tau)\right|^{2} d \tau \\
& =\min _{\omega \in \mathbf{R}_{0+}} \operatorname{Re} \widehat{G}(\mathbf{i} \omega) \int_{0}^{t}|u(\tau)|^{2} d \tau \geq 0 ; \quad \forall t \geq 0 .
\end{aligned}
$$

It has been proved, under zero initial conditions, that $\int_{0}^{t} y(\tau) u(\tau) d \tau \geq 0$ and $y(t) u(t) \geq 0 ; \forall t \geq 0$ and if $u \in L_{2}$ then $\int_{0}^{t} y(\tau) u(\tau) d \tau \geq \beta ; \forall t \geq 0$ for some $\beta \in[0, \infty)$ independent of $u$ (and independent of $t$ ). Since the zero state response generates and square-integrable output, since the input is square-integrable and since the zero input state is uniformly bounded as a result, the output is square-integrable for any square-integrable input. Also, the system is passive, since irrespective of the initial conditions, there exists some $\varepsilon \in \mathbf{R}$ such that $\int_{0}^{t} y(\tau) u(\tau) d \tau \geq \beta-\varepsilon^{\prime} \geq-\varepsilon$ since the initial conditions do not generate an unbounded homogeneous solution since $\widehat{G} \in \mathbf{R H}_{\infty}$ since $\widehat{G} \in\{\mathrm{PR}\}$. Property (i) has been proved. On the other hand, under any finite nonzero initial conditions $x_{0} \in \mathbf{R}^{n}$ :

$$
\begin{aligned}
& \int_{0}^{t} y(\tau) u(\tau) d \tau \geq \min _{\omega \in \mathbf{R}_{0+}} \operatorname{Re} \widehat{G}(\mathbf{i} \omega) \int_{0}^{t}|u(\tau)|^{2} d \tau \\
&-\left|\int_{0}^{t} \lambda\left(t-\tau, x_{0}\right) u(\tau) d \tau\right| ; \\
& \forall t \geq 0
\end{aligned}
$$

for some uniformly bounded $\lambda\left(t, x_{0}\right)$ since $\widehat{G}(s)$ is stable, (perhaps including eventual single critical poles) since it is in $\{\mathrm{PR}\}$. If, in addition, $\widehat{G} \in\{\mathrm{SPR}\}$ then it is strictly stable so that $\min _{\omega \in \mathbf{R}_{0+}} \operatorname{Re} \widehat{G}(\mathbf{i} \omega)>0$ and

$$
\begin{aligned}
&+\infty>\gamma \geq \int_{0}^{t} y(\tau) u(\tau) d \tau \\
& \geq \min _{\omega \in \mathbf{R}_{0+}} \operatorname{Re} \widehat{G}(\mathbf{i} \omega) \int_{0}^{t}|u(\tau)|^{2} d \tau \\
& \quad-\left|\int_{0}^{t} \lambda\left(t-\tau, x_{0}\right) u(\tau) d \tau\right| \\
&>\varepsilon_{u} \int_{0}^{t}|u(\tau)|^{2} d \tau-\varepsilon,
\end{aligned}
$$

since $u \in L_{2}$, for any time $t>0$, any given control and initial conditions, and some finite $\varepsilon_{u}>0$ and $\varepsilon \geq 0$ with $\lambda(t)=$ $c^{T} e^{A t} x_{0}$, where $c^{T}$ and $A$ are the output vector and matrix of dynamics of a state-space realization of initial state $x_{0}$ so that $\lambda(\cdot, 0)=0$. Property (ii) has been proved. Finally, if the system is externally positive and the input is nonnegative for all time then, for any given set of nonnegative initial conditions, one has that

$$
\begin{aligned}
+\infty> & \gamma \geq \int_{0}^{t} y(\tau) u(\tau) d \tau \\
\geq & \min _{\omega \in \mathbf{R}_{0+}} \operatorname{Re} \widehat{G}(\mathbf{i} \omega) \int_{0}^{t}|u(\tau)|^{2} d \tau \\
& +\int_{0}^{t} \lambda\left(t-\tau, x_{0}\right) u(\tau) d \tau \\
> & \varepsilon_{u} \int_{0}^{t}|u(\tau)|^{2} d \tau+\varepsilon \geq 0 ; \quad \forall t \geq 0
\end{aligned}
$$

which proves Property (iii). To prove Property (iv), note that $a_{G} \leq R_{\widehat{G}}=\left\|\left(\widehat{D}_{G}(\mathbf{i} \omega)-\widehat{N}_{G}(\mathbf{i} \omega)\right) /\left(\widehat{D}_{G}(\mathbf{i} \omega)+\widehat{N}_{G}(\mathbf{i} \omega)\right)\right\|_{\infty} \leq b_{G}$ holds if

$$
\begin{aligned}
& a_{G} \mid \operatorname{Re}\left(\widehat{D}_{G}(\mathbf{i} \omega)+\widehat{N}_{G}(\mathbf{i} \omega)\right) \\
& \quad+\mathbf{i} \operatorname{Im}\left(\widehat{D}_{G}(\mathbf{i} \omega)+\widehat{N}_{G}(\mathbf{i} \omega)\right) \mid
\end{aligned}
$$




$$
\begin{aligned}
& \leq \mid \operatorname{Re}\left(\widehat{D}_{G}(\mathbf{i} \omega)-\widehat{N}_{G}(\mathbf{i} \omega)\right) \\
& +\mathbf{i} \operatorname{Im}\left(\widehat{D}_{G}(\mathbf{i} \omega)-\widehat{N}_{G}(\mathbf{i} \omega)\right) \mid \\
& \leq b_{G} \mid \operatorname{Re}\left(\widehat{D}_{G}(\mathbf{i} \omega)+\widetilde{N}_{G}(\mathbf{i} \omega)\right) \\
& +\mathbf{i} \operatorname{Im}\left(\widehat{D}_{G}(\mathbf{i} \omega)+\widehat{N}_{G}(\mathbf{i} \omega)\right) \mid ; \quad \forall \omega \in \mathbf{R}_{0+}
\end{aligned}
$$

holds, that is, if

$$
\begin{aligned}
a_{G}^{2} & \left(\operatorname{Re}^{2}\left(\widehat{D}_{G}(\mathbf{i} \omega)+\widehat{N}_{G}(\mathbf{i} \omega)\right)\right. \\
& \left.+\operatorname{Im}^{2}\left(\widehat{D}_{G}(\mathbf{i} \omega)+\widehat{N}_{G}(\mathbf{i} \omega)\right)\right) \\
& \leq\left(\operatorname{Re}^{2}\left(\widehat{D}_{G}(\mathbf{i} \omega)-\widehat{N}_{G}(\mathbf{i} \omega)\right)\right. \\
& \left.+\operatorname{Im}^{2}\left(\widehat{D}_{G}(\mathbf{i} \omega)-\widehat{N}_{G}(\mathbf{i} \omega)\right)\right) \\
& \leq b_{G}^{2}\left(\operatorname{Re}^{2}\left(\widehat{D}_{G}(\mathbf{i} \omega)+\widehat{N}_{G}(\mathbf{i} \omega)\right)\right. \\
& \left.+\operatorname{Im}^{2}\left(\widehat{D}_{G}(\mathbf{i} \omega)+\widehat{N}_{G}(\mathbf{i} \omega)\right)\right) ; \quad \forall \omega \in \mathbf{R}_{0+}
\end{aligned}
$$

which leads to (6).

Remark 3. The generalization of Theorem 2 to the multiinput multioutput (MIMO) case (i.e., $m>1$ ) is direct by replacing the instantaneous power $y(t) u(t)$ by the scalar product $y^{T}(t) u(t)$ in the corresponding expressions.

The following two results discuss how the basic passivity property can become a stronger property as, for instance, strict input passivity or very strict passivity, by incorporating to the input-output operator a suitable parallel static inputoutput interconnection structure.

Proposition 4. Consider a class $[G]_{\rho D}$ of dynamic systems $G(\rho, D): \mathrm{H}_{e} \rightarrow \mathrm{H}_{e}$, defined as $G(\rho, D)=G_{0}+\rho D$ for given $\rho \in \mathbf{R}, D\left(\in \mathbf{R}^{m \times m}\right) \geq 0$ and $G_{0}: \mathrm{H}_{e} \rightarrow \mathrm{H}_{e}$, such that $G(\alpha, D) \in[G]_{\rho D}$ for any $\alpha \in[0, \rho]$. The following properties hold:

(i) Assume that $G(\rho, D)$ is very strictly passive, $D>0$ and $\varepsilon_{u \rho}>\rho \lambda_{\text {max }}\left(D+D^{T}\right) / 2$, where $\varepsilon_{u \rho}$ is the input passivity constant for $G(\rho, D)$. Then, $G(\alpha, D)$ is very strictly passive for all $\alpha \in[0, \rho]$. If $\varepsilon_{u \rho}=\rho \lambda_{\max }\left(D+D^{T}\right) / 2$ then $G(\alpha, D)$ is very strictly passive for all $\alpha \in(0, \rho]$ while $G_{0}$ is strictly output passive.

(ii) If $G_{0}$ is passive (resp., strictly output passive) then $G(\alpha, D)$ is strictly input passive (resp., very strictly passive) if $D>0$ for any $\alpha \in \mathbf{R}_{+}$.

Proof. Since $G(\alpha, D) \in[G]_{\rho D}$ for any given $\alpha \in[0, \rho], G_{0}=$ $G(0, D) \in[G]_{\rho D}$ (irrespective of $\left.D\right)$. Then, since $G(\rho, D)$ is very strictly passive $\exists \varepsilon_{\rho} \geq 0, \exists \varepsilon_{u \rho}>0$ and $\exists \varepsilon_{y \rho}>0$ s.t.

$$
\begin{array}{r}
\int_{0}^{t} y_{\rho}^{T}(\tau) u(\tau) d \tau=\int_{0}^{t}\left(G_{\rho} u\right)^{T}(\tau) u(\tau) d \tau \\
=\int_{0}^{t}\left(\left(G_{0} u\right)^{T}(\tau)+\rho u^{T}(\tau) D^{T}\right) u(\tau) d \tau
\end{array}
$$

$$
\begin{gathered}
\geq-\varepsilon_{\rho}+\varepsilon_{u \rho} \int_{0}^{t} u^{T}(\tau) u(\tau) d \tau \\
\quad+\varepsilon_{y \rho} \int_{0}^{t} y^{T}(\tau) y(\tau) d \tau
\end{gathered}
$$

for all $t \geq 0$ and any input-output pair $\left(u, y_{\alpha}\right)$ with input and output being defined from $\mathbf{R}_{0+}$ to $\mathbf{R}^{m}$ for any $\alpha \in[0, \rho]$. Thus,

$$
\begin{aligned}
& \int_{0}^{t} y_{0}^{T}(\tau) u(\tau) d \tau=\int_{0}^{t}\left(G_{0} u\right)^{T}(\tau) u(\tau) \geq-\varepsilon_{\rho} \\
& +\int_{0}^{t} u^{T}(\tau)\left(\varepsilon_{u \rho} I_{m}-\rho D^{T}\right) u(\tau) d \tau+\varepsilon_{y \rho} \int_{0}^{t} y^{T}(\tau) \\
& \cdot y(\tau) d \tau \geq-\varepsilon_{\rho}+\int_{0}^{t} u^{T}(\tau) \\
& \cdot\left(\varepsilon_{u \rho}-\frac{\rho}{2} \lambda_{\max }\left(D+D^{T}\right)\right) u^{T}(\tau) u(\tau) d \tau \\
& +\varepsilon_{y \rho} \int_{0}^{t} y^{T}(\tau) y(\tau) d \tau=-\varepsilon_{\rho}+\varepsilon_{u 0} \int_{0}^{t} u^{T}(\tau) \\
& \cdot u(\tau) d \tau+\varepsilon_{y \rho} \int_{0}^{t} y^{T}(\tau) y(\tau) d \tau ; \quad \forall t \geq 0
\end{aligned}
$$

where $\varepsilon_{u 0}=\varepsilon_{u \rho}-(\rho / 2) \lambda_{\max }\left(D+D^{T}\right)>0$ since $D>0$ and $\varepsilon_{u \rho}>\rho \lambda_{\text {max }}\left(D+D^{T}\right) / 2$. Then, $G_{0}$ is very strictly passive with passivity, input passivity, and output passivity constants $\varepsilon_{0}=$ $\varepsilon_{\rho} \geq 0, \varepsilon_{u 0}=\varepsilon_{u \rho}-(\rho / 2) \lambda_{\max }\left(D+D^{T}\right)>0$, and $\varepsilon_{y 0}=\varepsilon_{y \rho}>0$. If $\varepsilon_{u \rho}=\rho \lambda_{\max }\left(D+D^{T}\right) / 2$ then $G_{0}$ is guaranteed to be just output-strictly passive. Now, for any $\alpha \in[0, \rho]$, one has

$$
\begin{aligned}
& \int_{0}^{t} y_{\alpha}^{T}(\tau) u(\tau) d \tau=\int_{0}^{t}\left(G_{\alpha} u\right)^{T}(\tau) u(\tau) d \tau \\
& \quad=\int_{0}^{t}\left(\left(G_{0} u\right)^{T}(\tau)+\alpha u^{T}(\tau) D^{T}\right) u(\tau) d \tau \\
& \quad=\int_{0}^{t}\left(y_{0}^{T}(\tau) u(\tau)+\alpha u^{T}(\tau) D^{T} u(\tau)\right) d \tau \\
& \geq-\varepsilon_{\rho}+\int_{0}^{t} u^{T}(\tau) \\
& \quad \cdot\left(\varepsilon_{u \rho}+\frac{\alpha}{2} \lambda_{\min }\left(D+D^{T}\right)-\frac{\rho}{2} \lambda_{\max }\left(D+D^{T}\right)\right) \\
& \quad \cdot u^{T}(\tau) u(\tau) d \tau+\varepsilon_{y \rho} \int_{0}^{t} y^{T}(\tau) y(\tau) d \tau \\
& \geq-\varepsilon_{\rho}+\int_{0}^{t} u^{T}(\tau)\left(\varepsilon_{u \rho}-\frac{\rho}{2} \lambda_{\max }\left(D+D^{T}\right)\right) u^{T}(\tau) \\
& \quad \cdot u(\tau) d \tau+\varepsilon_{y \rho} \int_{0}^{t} y^{T}(\tau) y(\tau) d \tau ;
\end{aligned}
$$

$\forall t \geq 0$. Then, $G(\alpha, D)$ is very strictly passive with passivity, input passivity, and output passivity constants $\varepsilon_{\alpha}=\varepsilon_{\rho} \geq 0$, $\varepsilon_{u \alpha}=\varepsilon_{u \rho}>0$ and $\varepsilon_{y \alpha}=\varepsilon_{y \rho}>0$ for all $\alpha \in[0, \rho]$ if 
$\varepsilon_{u \rho}>\rho \lambda_{\max }\left(D+D^{T}\right) / 2$ while $\varepsilon_{u \rho}=\rho \lambda_{\max }\left(D+D^{T}\right) / 2$ implies $\varepsilon_{u 0}=\varepsilon_{u \rho}-(\rho / 2) \lambda_{\max }\left(D+D^{T}\right)=0$ so that $G_{0}$ is strictly output passive and $G(\alpha, D)$ is very strictly passive for $\alpha \in(0, \rho]$. Property (i) has been proved. Property (ii) follows from (15a) with $\varepsilon_{u \alpha}=\alpha$.

Proposition 5. Assume that $G_{0}$ is passive and nonexpansive. Then:

(i) $G_{\rho}=G_{0}+\rho D$ is $L_{2}$-stable and strictly input passive if $\rho \in \mathbf{R}_{+}$,

(ii) $G_{0}$ is $L_{2}$-stable if $\rho>-2\left(\lambda_{0}+\gamma_{0}^{2}\right) /\left(\lambda_{\max }\left(D+D^{T}\right)-\right.$ $\left.\lambda_{\text {min }}\left(D+D^{T}\right)\right)$ if $D\left(\neq I_{m}, 0\right) \geq 0$,

(iii) $G_{0}$ is $L_{2}$-stable if $D=I_{m}$ for any given $\rho \in \mathbf{R}$.

Proof. Since $G_{0}$ is passive and nonexpansive, one has

$$
\begin{aligned}
\lambda_{0} & +\gamma_{0}^{2} \int_{0}^{t} u^{T}(\tau) u(\tau) d \tau \\
& +\frac{\rho}{2} \lambda_{\max }\left(D+D^{T}\right) \int_{0}^{t} u^{T}(\tau) u(\tau) d \tau \\
& \geq \int_{0}^{t}\left(G_{\rho} u\right)^{T}(\tau) u(\tau) d \tau \\
& =\int_{0}^{t}\left(\left(G_{0}+\rho D\right) u\right)^{T}(\tau) u(\tau) d \tau \\
& \geq \int_{0}^{t}\left(G_{0} u\right)^{T}(\tau) u(\tau) d \tau \\
& +\frac{\rho}{2} \lambda_{\min }\left(D+D^{T}\right) \int_{0}^{t} u^{T}(\tau) u(\tau) d \tau \geq-\varepsilon_{0} \\
& +\frac{\rho}{2} \lambda_{\min }\left(D+D^{T}\right) \int_{0}^{t} u^{T}(\tau) u(\tau) d \tau ; \quad \forall t \geq 0 .
\end{aligned}
$$

Thus, if $u \in L_{2}^{m}$ then

$$
\begin{aligned}
& +\infty>M=\lambda_{0}+\left(\gamma_{0}^{2}+\frac{\rho}{2} \lambda_{\max }\left(D+D^{T}\right)\right) \\
& \cdot \int_{0}^{t} u^{T}(\tau) u(\tau) d \tau \geq \int_{0}^{t}\left(G_{\rho} u\right)^{T}(\tau) u(\tau) d \tau \geq-\varepsilon_{0} \\
& +\frac{\rho}{2} \lambda_{\min }\left(D+D^{T}\right) \int_{0}^{t} u^{T}(\tau) u(\tau) d \tau ; \quad \forall t \geq 0 . \\
& +\infty>M_{0}=\lambda_{0} \\
& +\left(\gamma_{0}^{2}+\frac{\rho}{2}\left[\lambda_{\max }\left(D+D^{T}\right)-\lambda_{\min }\left(D+D^{T}\right)\right]\right) \\
& \cdot \int_{0}^{t} u^{T}(\tau) u(\tau) d \tau \geq \int_{0}^{t}\left(G_{0} u\right)^{T}(\tau) u(\tau) d \tau \geq-\varepsilon_{0} ;
\end{aligned}
$$

$\forall t \geq 0$.

Thus, $G_{\rho}$ is $L_{2}$-stable and strictly input passive if $\rho \in \mathbf{R}_{+} ; G_{0}$ is $L_{2}$-stable if $\rho>-2\left(\lambda_{0}+\gamma_{0}^{2}\right) /\left(\lambda_{\max }\left(D+D^{T}\right)-\lambda_{\text {min }}(D+\right.$ $\left.\left.D^{T}\right)\right)$ if $D\left(\neq I_{m}, 0\right) \geq 0$ since the maximum and minimum eigenvalues of $D+D^{T}$ are distinct. If $D=I_{m}$ then $M_{0}=$ $\lambda_{0}+\gamma_{0}^{2}>0$ for any real $\rho \in \mathbf{R}$ and $G_{0}$ is $L_{2}$-stable.

Remark 6. It turns out through simple mathematical derivations that Propositions 1-2 still hold under the replacement $D \rightarrow G_{1}$, where $G_{1}: \mathrm{H}_{e} \rightarrow \mathrm{H}_{e}$ is passive with associated constant $\varepsilon_{1} \leq 0$ for the properties to be extended from the case that $D \geq 0$ and strictly input passive for those extended from the case when $D>0$.

\section{Feed-Forward and Feedback Controllers and Closed-Loop Passivity}

It is now discussed how the passivity properties can be improved via feedback with respect to an external reference input signal. Consider the following linear time-invariant SISO cases:

(a) The controlled plant transfer function $\widehat{G}(s)$, whose relative passivity index [Theorem 2 (iv)] is $R_{\widehat{G}}=\sup _{\omega \in \mathbf{R}_{0+}} \mid(1-$ $\widehat{G}(\mathbf{i} \omega)) /(1+\widehat{G}(\mathbf{i} \omega)) \mid$, is controlled by a feedback controller of transfer function $\widehat{K}_{1}(s)$ so that

$$
\widehat{M}_{1}(s)=\frac{1-\widehat{T}_{1}(s)}{1+\widehat{T}_{1}(s)}=\frac{1+\widehat{G}(s)\left(\widehat{K}_{1}(s)-1\right)}{1+\widehat{G}(s)\left(\widehat{K}_{1}(s)+1\right)},
$$

where $\widehat{T}_{1}(s)=\widehat{G}(s) /\left(1+\widehat{G}(s) \widehat{K}_{1}(s)\right)$ is the resulting closedloop transfer function. The closed-loop relative passivity index is $R_{\widehat{T}_{1}}=\sup _{\omega \in \mathbf{R}_{0+}}\left|\left(1-\widehat{T}_{1}(\mathbf{i} \omega)\right) /\left(1+\widehat{T}_{1}(\mathbf{i} \omega)\right)\right|=$ $\sup _{\omega \in \mathbf{R}_{0+}}\left|\widehat{M}_{1}(\mathbf{i} \omega)\right|$. For any given $\widehat{T}_{1}(s)$ and associated $\widehat{M}_{1}(s)$, the controller transfer function is

$$
\begin{aligned}
\widehat{K}_{1}(s) & =\frac{(2 \widehat{G}(s)-1) \widehat{T}_{1}(s)+1}{2 \widehat{G}(s) \widehat{T}_{1}(s)} \\
& =\frac{\widehat{G}(s)\left(1+\widehat{M}_{1}(s)\right)+\widehat{M}_{1}(s)}{\widehat{G}(s)\left(1-\widehat{M}_{1}(s)\right)} .
\end{aligned}
$$

(b) The controlled plant transfer function $\widehat{G}(s)$ is controlled by a feed-forward controller of transfer function $\widehat{K}_{2}(s)$ so that

$$
\widehat{M}_{2}(s)=\frac{1-\widehat{T}_{2}(s)}{1+\widehat{T}_{2}(s)}=\frac{1}{1+2 \widehat{G}(s) \widehat{K}_{2}(s)},
$$

where $\widehat{T}_{2}(s)=\widehat{G}(s) \widehat{K}_{2}(s) /\left(1+\widehat{G}(s) \widehat{K}_{2}(s)\right)$ is the resulting closed-loop transfer function. The closed-loop relative passivity index is $R_{\widehat{T}_{2}}=\sup _{\omega \in \mathbf{R}_{0+}}\left|\left(1-\widehat{T}_{2}(\mathbf{i} \omega)\right) /\left(1+\widehat{T}_{2}(\mathbf{i} \omega)\right)\right|=$ $\sup _{\omega \in \mathbf{R}_{0+}}\left|\widehat{M}_{2}(\mathbf{i} \omega)\right|$. For any given $\widehat{T}_{1}(s)$ and associated $\widehat{M}_{1}(s)$, the controller transfer function is

$$
\widehat{K}_{2}(s)=\frac{\widehat{T}_{2}(s)}{\widehat{G}(s)\left(1-\widehat{T}_{2}(s)\right)}=\frac{1-\widehat{M}_{2}(s)}{2 \widehat{G}(s) \widehat{M}_{2}(s)} .
$$

The subsequent result uses the above considerations to rely on the property of linear time-invariant systems establishing that a positive real transfer function can be designed by using 
feedback or feed-forward control laws for the case when the plant transfer function is inversely stable even if it is not positive real or stable.

Theorem 7. Assume that $\widehat{G}(s)$ is inversely stable with relative degree 0 or 1 while nonnecessarily in $\{P R\}$ (or even nonnecessarily in $\mathbf{R H}_{\infty}$ ). Then, the following properties hold:

(i) A nonunique (state-space) realizable closed-loop transfer function $\widehat{T}_{1} \in\{P R\}$, or, respectively, $\widehat{T}_{1} \in\{S P R\}$, may be designed via a stable feedback controller of transfer function $\widehat{K}_{1}(s)$ (19) which is realizable if $\widehat{G}(s)$ and $\widehat{T}_{1}(s)$ have zero relative degree. In the above cases, $\widehat{T}_{1}^{-1} \in\{P R\}$, or, respectively, $\widehat{T}_{1}^{-1} \in$ $\{S P R\}$.

(ii) A nonunique realizable closed-loop transfer function $\widehat{T}_{2} \in\{P R\}$, or $\widehat{T}_{2} \in\{S P R\}$, may be designed via a feed-forward controller of transfer function $\widehat{K}_{2}(s)$ via (21) which is realizable if the relative degree of the closed-loop transfer function $\widehat{T}_{2}(s)$ is not less than that of the plant transfer function $\widehat{G}(s)$. In the above cases, $\widehat{T}_{2}^{-1} \in\{P R\}$, or, respectively, $\widehat{T}_{2}^{-1} \in\{S P R\}$.

Proof. A nonunique realizable closed-loop transfer function can be targeted as design objective through a feedback controller of transfer function $\widehat{K}_{1}(s)$, (19), such that $R_{\widehat{T}_{1}} \leq 1$. Since $R_{\widehat{T}_{1}} \leq 1$, it turns out that $\widehat{M}_{1}(s)$ is bounded real (i.e., Schur, i.e., with $H_{\infty}$-norm not exceeding unity and with real numerator and denominator coefficients) so that $\widehat{T}_{1} \in\{\mathrm{PR}\}$ (since $\widehat{T}_{1} \in\{\mathrm{PR}\}$ if and only if $\widehat{M}_{1}(s)$ is bounded real, [4]) then with a relative degree ( 0 or 1 ) being identical to that of $\widehat{G}(s)$. The controller is realizable if $\widehat{G}(s)$ and $\widehat{T}_{1}(s)$ have zero relative degree. Then, $\widehat{T}_{1}(s)$ is stable and inversely stable. Since $\widehat{G}(s)$ is inversely stable and $\widehat{T}_{1}^{-1}(s)$ is stable, since $\widehat{T}_{1}^{-1} \in\{\mathrm{PR}\}$ since $\widehat{T}_{1} \in\{\mathrm{PR}\}$, then it follows from (18)-(19) that $\widehat{K}_{1} \in \mathbf{R H}_{\infty}$. Also, $\widehat{T}_{1}(s) \in\{\mathrm{SPR}\}$ if and only if $R_{\widehat{T}_{1}}<1$, that is, if and only if $\widehat{M}_{1}(s)$ is strictly bounded real. Then, $\widehat{T}_{1}$ is strictly stable and inversely strictly stable. Property (i) has been proved. Property (i) is proved in a similar way via (20)-(21) with the controller realizability constraint $\operatorname{deg}\left(D_{2}\right) \geq \operatorname{deg}(D)+$ $\operatorname{deg}\left(N_{2}\right)-\operatorname{deg}(N)$; that is, the relative degree of the closedloop transfer function is not less than that of that of the plant $\widehat{G}(s)$.

In the light of Propositions 4 and 5 and Remark 6, it turns out that real positivity of a time-invariant system can be achieved by modifying a stable transfer matrix with the incorporation of an input-output interconnection gain being at least positive semidefinite. Similar conclusions follow by the use of close arguments to those in Theorem 7 on the inverse of a transfer matrix $\widehat{G}(s)$ to achieve positive real closed-loop transfer matrices under appropriate feedback and feed-forward controllers. The results can be extended to the discrete case [13]. The subsequent result follows related to these comments:

Theorem 8. The following properties hold:

(i) Assume that $\widehat{G} \in \mathbf{R H}_{\infty}$ is a transfer matrix of order $m \times m$. Then, $\widehat{G}_{1} \in\{P R\}$, where $\widehat{G}_{1}(s)=\widehat{G}(s)+D$ with
$D(\succeq 0) \in \mathbf{R}^{m \times m}$ if $\widehat{G}(\mathbf{i} \omega)+\widehat{G}^{T}(-\mathbf{i} \omega) \succeq-\left(D+D^{T}\right) ; \forall \omega \in \mathbf{R}_{0+}$. If $m=1$ then the condition becomes $\operatorname{Re} \widehat{G}(\mathbf{i} \omega) \geq-d ; \forall \omega \in \mathbf{R}_{0+}$ with $d=D$. If $\widehat{G}(\mathbf{i} \omega)+\widehat{G}^{T}(-\mathbf{i} \omega)>-\left(D+D^{T}\right) ; \forall \omega \in \mathbf{R}_{0+}$ and then $\widehat{G}_{1} \in\{S P R\}$ which becomes $\operatorname{Re} \widehat{G}(\mathbf{i} \omega)>-d$ if $m=1$; $\forall \omega \in \mathbf{R}_{0+}$.

(ii) Assume that $\widehat{G}(s)$ is an inversely stable transfer matrix order $m \times m$ controlled by a linear time-invariant feedback controller of transfer matrix $\widehat{K}_{1}(s)$ of order $m \times m$. Then, $\widehat{T}_{1}, \widehat{T}_{1}^{-1} \in$ $\{P R\}$ (resp., $\widehat{T}_{1}, \widehat{T}_{1}^{-1} \in\{S P R\}$ ) if

$$
\begin{aligned}
& \inf _{\omega \in \mathbf{R}_{0+}} \lambda_{\min }\left[\widehat{K}_{1}(\mathbf{i} \omega)+\widehat{K}_{1}(-\mathbf{i} \omega)\right] \\
& \quad \geq-\sup _{\omega \in \mathbf{R}_{0+}} \lambda_{\max }\left(\widehat{G}^{-1}(\mathbf{i} \omega)+\widehat{G}^{-T}(-\mathbf{i} \omega)\right)
\end{aligned}
$$

(resp., the above inequality is strict).

(iii) Assume that $\widehat{G}(s)$ is an inversely stable transfer matrix order $m \times m$ being controlled by a linear time-invariant feedforward controller of transfer matrix $\widehat{K}_{2}(s)$ of order $m \times$ m. Then, $\widehat{T}_{2}, \widehat{T}_{2}^{-1} \in\{P R\}$ (resp., $\widehat{T}_{2}, \widehat{T}_{2}^{-1} \in\{S P R\}$ ) if $\inf _{\omega \in \mathbf{R}_{0+}} \lambda_{\text {min }}\left[\widehat{K}_{2}(\mathbf{i} \omega) \widehat{G}^{-1}(\mathbf{i} \omega)+\widehat{G}^{-T}(\mathbf{i} \omega) \widehat{K}_{2}^{T}(-\mathbf{i} \omega)\right] \geq-1$ (resp., the inequality is strict).

Proof. The proof of Property (i) is direct from the conditions of positive and strictly positive realness for $\widehat{G}_{1}(s)$. Inspired by the definitions of positive realness and Theorem 7 for the SISO case, Properties (ii)-(iii) are proved as follows. By using the feedback and feed-forward controllers, the following respective closed-loop transfer matrices are obtained:

$$
\begin{aligned}
\widehat{T}_{1}(s) & =\left(I_{m}+\widehat{G}(s) \widehat{K}_{1}(s)\right)^{-1} \widehat{G}(s) \\
& =\left[\widehat{G}^{-1}(s)\left(I_{m}+\widehat{G}(s) \widehat{K}_{1}(s)\right)\right]^{-1} \\
& =\left[\widehat{G}^{-1}(s)+\widehat{K}_{1}(s)\right]^{-1}, \\
\widehat{T}_{2}(s) & =\left(I_{m}+\widehat{G}(s) \widehat{K}_{2}(s)\right)^{-1} \widehat{G}(s) \widehat{K}_{2}(s) \\
& =\left[\widehat{K}_{2}^{-1}(s) \widehat{G}^{-1}(s)\left(I_{m}+\widehat{G}(s) \widehat{K}_{2}(s)\right)\right]^{-1} \\
& =\left[I_{m}+\widehat{K}_{2}^{-1}(s) \widehat{G}^{-1}(s)\right]^{-1}
\end{aligned}
$$

with inverses

$$
\begin{aligned}
& \widehat{T}_{1}^{-1}(s)=\widehat{G}^{-1}(s)+\widehat{K}_{1}(s) ; \\
& \widehat{T}_{2}^{-1}(s)=I_{m}+\widehat{K}_{2}^{-1}(s) \widehat{G}^{-1}(s) .
\end{aligned}
$$

Then, $\widehat{T}_{1}(s)$ and $\widehat{T}_{1}^{-1}(s)$ (resp., $\widehat{T}_{2}(s)$ and $\left.\widehat{T}_{2}^{-1}(s)\right)$ are positive real if

$$
\begin{array}{r}
\widehat{K}_{1}(\mathbf{i} \omega)+\widehat{K}_{1}^{T}(-\mathbf{i} \omega) \succeq-\left(\widehat{G}^{-1}(\mathbf{i} \omega)+\widehat{G}^{-T}(-\mathbf{i} \omega)\right) \\
\forall \omega \in \mathbf{R}_{0+}
\end{array}
$$


which is guaranteed if $\inf _{\omega \in \mathbf{R}_{0+}} \lambda_{\min }\left[\widehat{K}_{1}(\mathbf{i} \omega)+\widehat{K}_{1}(-\mathbf{i} \omega)\right] \geq$ $-\sup _{\omega \in \mathbf{R}_{0+}} \lambda_{\max }\left(\widehat{G}^{-1}(\mathbf{i} \omega)+\widehat{G}^{-T}(-\mathbf{i} \omega)\right)$, respectively, if

$$
\begin{aligned}
\widehat{K}_{2}^{-1}(\mathbf{i} \omega) \widehat{G}^{-1}(\mathbf{i} \omega)+\widehat{G}^{-T}(-\mathbf{i} \omega) \widehat{K}_{2}^{-T}(-\mathbf{i} \omega) & \geq-I_{m} ; \\
& \forall \omega \in \mathbf{R}_{0+}
\end{aligned}
$$

which is guaranteed if $\inf _{\omega \in \mathbf{R}_{0+}} \lambda_{\min }\left[\widehat{K}_{2}(\mathbf{i} \omega) \widehat{G}^{-1}(\mathbf{i} \omega)+\right.$ $\left.\widehat{G}^{-T}(\mathbf{i} \omega) \widehat{K}_{2}^{T}(-\mathbf{i} \omega)\right] \geq-1$. Strict positive realness in each of both cases is guaranteed under the corresponding strict inequalities in (26)-(27).

Note that a sufficient condition for (26) to hold for the SISO case (i.e., $m=1$ ) is $\min _{\omega \in \mathbf{R}_{0+}} \operatorname{Re} \widehat{K}_{1}(\mathbf{i} \omega) \geq$ $-d_{\widehat{G}} / \sup _{\omega \in \mathbf{R}_{0+}}\|\widehat{G}\|_{\infty}^{2}$, where $\operatorname{Re} \widehat{G}(\mathbf{i} \omega) \geq-d_{\widehat{G}}$, if $\widehat{G}(s)$ is stable and realizable (so that its $H_{\infty}$ norm exists) and $\min _{\omega \in \mathbf{R}_{0+}} \operatorname{Re} \widehat{K}_{1}(\mathbf{i} \omega) \geq-d_{\widehat{G}} / \sup _{\omega \in \mathbf{R}_{0+}}\|\widehat{G}\|_{\infty}^{2}$.

The proof of Theorem 8(i) can be also addressed from the fact that the inverse of a positive real matrix is positive real and the subsequent derivations if $D>0$ :

$$
\begin{aligned}
\widehat{G}^{-1}(s) & =(\widehat{G}(s)+D)^{-1}=\left[D\left(I_{m}+D^{-1} \widehat{G}(s)\right)\right]^{-1} \\
& =\left(I_{m}+D^{-1} \widehat{G}(s)\right)^{-1} D^{-1}
\end{aligned}
$$

and $\widehat{G}^{-1} \in\{\mathrm{PR}\}$, then $\widehat{G} \in\{\mathrm{PR}\}$, if

$$
\begin{aligned}
& \left(I_{m}+D^{-1} \widehat{G}(\mathbf{i} \omega)\right)^{-1} D^{-1}+D^{-T}\left(I_{m}+D^{-1} \widehat{G}(\mathbf{i} \omega)\right)^{-T} \\
& \quad \geq 0 ; \quad \forall \omega \in \mathbf{R}_{0+} .
\end{aligned}
$$

Since $I_{m}+D^{-1} \widehat{G}(\mathbf{i} \omega)>0, \forall \omega \in \mathbf{R}_{0+}$, the above matrix relation is equivalent to

$$
\begin{aligned}
D^{-1} & +\left(I_{m}+D^{-1} \widehat{G}(\mathbf{i} \omega)\right) D^{-T}\left(I_{m}+D^{-1} \widehat{G}(\mathbf{i} \omega)\right)^{-T} \\
\geq & 0 ; \quad \forall \omega \in \mathbf{R}_{0+}
\end{aligned}
$$

and to

$$
\begin{aligned}
D^{-1}\left(I_{m}+D^{-1} \widehat{G}(\mathbf{i} \omega)\right)^{T}+\left(I_{m}+D^{-1} \widehat{G}(\mathbf{i} \omega)\right) D^{-T} & \geq 0 ; \\
\forall \omega & \in \mathbf{R}_{0+}
\end{aligned}
$$

so that

$$
\begin{aligned}
& D^{-1}\left(\widehat{G}(\mathbf{i} \omega)+\widehat{G}^{T}(\mathbf{i} \omega)\right) D^{-T} \geq-\left(D^{-1}+D^{-T}\right) ; \\
& \forall \omega \in \mathbf{R}_{0+}
\end{aligned}
$$

which yields, equivalently, $\widehat{G}(\mathbf{i} \omega)+\widehat{G}^{T}(-\mathbf{i} \omega) \geq-\left(D+D^{T}\right)$; $\forall \omega \in \mathbf{R}_{0+}$.

\section{Regenerative versus Passive Systems}

Note that passive systems are intrinsically stable and either consume or dissipate energy for all time. Looking at Definition 1(3), we can give an opposed one as follows:
Definition 9. A dynamic system is nonpassive (or active or, so-called, regenerative) if $\int_{0}^{t_{i}} y^{T}(\tau) u(\tau) d \tau+\varepsilon_{t_{i}}<0$ for some unbounded sequences $E=\left\{\varepsilon_{t_{i}}\right\} \subseteq \mathbf{R}_{0+}, T=\left\{t_{i}\right\} \subseteq \mathbf{R}_{0+}$ which satisfy the conditions

(1) $0<\delta_{i-1} \leq t_{i+1}-t_{i} \leq \delta_{i}<\infty ; \forall i \in \mathbf{Z}_{0+}$ for some positive bounded sequence $\Delta=\left\{\delta_{i}\right\}$,

(2) $0<\theta_{i-1} \leq \widetilde{\varepsilon}_{t_{i}}=\varepsilon_{t_{i+1}}-\varepsilon_{t_{i}} \leq \theta_{i}<\infty ; \forall i \in \mathbf{Z}_{0+}$ for some positive bounded sequence $\Theta=\left\{\delta_{i}\right\}$,

(3) $\varepsilon_{i}, t_{i} \rightarrow+\infty$ as $i \rightarrow+\infty$.

The following result follows for a nonpassive system.

Theorem 10. If a dynamic system is nonpassive then $\lim _{t \rightarrow \infty} \int_{0}^{t} y^{T}(\tau) u(\tau) d \tau=-\infty$.

Proof. Define $\widetilde{\varepsilon}_{t_{i}} \in \mathbf{R}_{+}$such that $\int_{0}^{t_{i}} y^{T}(\tau) u(\tau) d \tau=-\varepsilon_{t_{i}}-\widetilde{\varepsilon}_{t_{i}}<$ $-\varepsilon_{t_{i}}$. Thus,

$$
\begin{aligned}
\int_{t_{i}}^{t_{i+1}} y^{T}(\tau) u(\tau) d \tau= & -\varepsilon_{t_{i+1}}-\widetilde{\varepsilon}_{t_{i+1}} \\
& -\int_{0}^{t_{i}} y^{T}(\tau) u(\tau) d \tau \\
= & \varepsilon_{t_{i}}+\widetilde{\varepsilon}_{t_{i}}-\left(\varepsilon_{t_{i+1}}+\widetilde{\varepsilon}_{t_{i+1}}\right), \\
\int_{0}^{t} y^{T}(\tau) u(\tau) d \tau= & \int_{0}^{t_{i}} y^{T}(\tau) u(\tau) d \tau \\
& +\int_{t_{i}}^{t} y^{T}(\tau) u(\tau) d \tau \\
= & -\varepsilon_{t_{i}}-\widetilde{\varepsilon}_{t_{i}}+\int_{t_{i}}^{t} y^{T}(\tau) u(\tau) d \tau, \\
\int_{0}^{t} y^{T}(\tau) u(\tau) d \tau= & \int_{0}^{t_{i+1}} y^{T}(\tau) u(\tau) d \tau \\
& -\int_{t}^{t_{i+1}} y^{T}(\tau) u(\tau) d \tau \\
= & -\varepsilon_{t_{i+1}}-\widetilde{\varepsilon}_{t_{i+1}} \\
& -\int_{t}^{t_{i+1}} y^{T}(\tau) u(\tau) d \tau .
\end{aligned}
$$

Subtracting the two above ones:

$$
\begin{gathered}
\varepsilon_{t_{i+1}}-\varepsilon_{t_{i}}+\left|\widetilde{\varepsilon}_{t_{i+1}}-\widetilde{\varepsilon}_{t_{i}}\right| \geq\left|\int_{t_{i}}^{t_{i+1}} y^{T}(\tau) u(\tau) d \tau\right| \geq 2 \\
\cdot \max \left(\left|\int_{t}^{t_{i+1}} y^{T}(\tau) u(\tau) d \tau\right|,\left|\int_{t_{i}}^{t} y^{T}(\tau) u(\tau) d \tau\right|\right), \\
\left|\varepsilon_{t_{i}}+\widetilde{\varepsilon}_{t_{i}}-\varepsilon_{t_{i+1}}+\varepsilon_{t_{i}}-\right| \widetilde{\varepsilon}_{t_{i+1}}-\widetilde{\varepsilon}_{t_{i}}|| \leq \mid \varepsilon_{t_{i}}+\widetilde{\varepsilon}_{t_{i}} \\
-\left|\int_{t_{i}}^{t} y^{T}(\tau) u(\tau) d \tau\right||\leq| \int_{0}^{t} y^{T}(\tau) u(\tau) d \tau \mid \leq-\varepsilon_{t_{i}}
\end{gathered}
$$




$$
\begin{aligned}
& -\widetilde{\varepsilon}_{t_{i}}+\left|\int_{t_{i}}^{t} y^{T}(\tau) u(\tau) d \tau\right| \leq-\left(\varepsilon_{t_{i}}+\widetilde{\varepsilon}_{t_{i}}\right) \\
& +\frac{1}{2\left(\varepsilon_{t_{i+1}}-\varepsilon_{t_{i}}+\left|\widetilde{\varepsilon}_{t_{i+1}}-\widetilde{\varepsilon}_{t_{i}}\right|\right)}
\end{aligned}
$$

since $\left\{\varepsilon_{t_{i}}\right\}$ is unbounded but its associated incremental sequence $\left\{\widetilde{\varepsilon}_{t_{i}}\right\}$ is bounded, $\left\{2 \varepsilon_{t_{i}}-\varepsilon_{t_{i+1}}\right\} \rightarrow \infty$ as $t_{i} \rightarrow \infty$; then $\left|\int_{0}^{\infty} y^{T}(\tau) u(\tau) d \tau\right|<+\infty$ contradicts the above relations.

Remark 11. Note that a nonpassive system can reach an absolute infinity energy measure in finite time under certain atypical inputs as, for instance, a second-order impulsive Dirac input of appropriate component signs at some time instant $t_{1}<\infty$ with $u(t)=0$ for $t>t_{1}$. Then, $\int_{0}^{t_{1}} y^{T}(\tau) u(\tau) d \tau=\lim _{t \rightarrow \infty} \int_{0}^{t} y^{T}(\tau) u(\tau) d \tau=-\infty$

The following result is concerned with passive versus nonpassive dynamic systems.

\section{Theorem 12. The following properties hold:}

(i) A passive system cannot be nonpassive in any time subinterval. A nonpassive system in some time interval cannot be a passive system.

(ii) A passive system is always stable and also dissipative (i.e., the dissipative energy function takes nonnegative values for all time) including the conservative particular case implying identically zero dissipation through time.

(iii) A nonpassive system can be stable or unstable (so, stable systems are nonnecessarily passive).

Proof. Property (i) is a direct consequence of Definitions 1(3) and 9 and Remark 11 since if the system is nonpassive so that it satisfies the constraint of Definition 9, it cannot satisfy a reversed passivity condition (for all time) of Definition 1(3) since $\lim _{t \rightarrow \infty} \int_{0}^{t} y^{T}(\tau) u(\tau) d \tau=-\infty$ is not compatible with the passivity condition. The converse statement is direct. We now prove Properties [(ii)-(iii)]. Note from Definition 9 that if $V: \mathbf{R}^{n} \times \mathbf{R}_{0+} \rightarrow \mathbf{R}_{0+}$ is an energy measure storage state function, as for instance a Lyapunov function, and if the system is passive (resp., nonpassive) then there exists $\varepsilon \in \mathbf{R}_{0+}$, respectively; there exists some unbounded sequences $E=$ $\left\{\varepsilon_{t_{i}}\right\} \subseteq \mathbf{R}_{0+}, T=\left\{t_{i}\right\} \subseteq \mathbf{R}_{0+}$ and

$$
\int_{0}^{t} y^{T}(\tau) u(\tau) d \tau=V(x(t))-V(x(0))+d(t) \geq-\varepsilon
$$

$$
\forall t \in \mathbf{R}_{0+} \text {, }
$$

respectively,

$$
\begin{aligned}
\int_{0}^{t_{i}} y^{T}(\tau) u(\tau) d \tau & =V(x(t))-V(x(0))+d(t) \\
& <-\varepsilon_{t_{i}}
\end{aligned}
$$

with $E=\left\{\varepsilon_{t_{i}}\right\} \subseteq \mathbf{R}_{0+}, T=\left\{t_{i}\right\} \subseteq \mathbf{R}_{0+}$, where $d(t)$ is the dissipation function and $\int_{0}^{t} y^{T}(\tau) u(\tau) d \tau ; \forall t \in \mathbf{R}_{0+}$ is interpreted as the external environment supplied or consumed energy on the interval $[0, t]$. Thus, the following cases are of interest.

Case 1 (passive system). One has from (23) that

$$
\begin{aligned}
d(t) & =\int_{0}^{t} y^{T}(\tau) u(\tau) d \tau+V(0)-V(t) \\
& \geq \max (V(0)-(\varepsilon+V(t)), 0) \geq 0 ; \quad \forall t \in \mathbf{R}_{0+}
\end{aligned}
$$

is a condition of energy dissipation for all time which happens if either

$$
\begin{gathered}
([V(0)-\varepsilon-V(t) \geq 0] \Longleftrightarrow \\
[V(t) \leq V(0)-\varepsilon]) \Longrightarrow \\
{[(\varepsilon=0) \wedge(V(t) \leq V(0))]}
\end{gathered}
$$

for all $t \in \mathbf{R}_{0+}$ and $d(t) \geq \int_{0}^{t} y^{T}(\tau) u(\tau) d \tau$ for all $t \in \mathbf{R}_{0+}$ which is also coherent with a null energetic interchange with the environment or if for all $t \in \mathbf{R}_{0+}$

$$
\begin{gathered}
{[0>V(0)-\varepsilon-V(t)] \Longleftrightarrow} \\
{[V(t)>V(0)-\varepsilon] .}
\end{gathered}
$$

Then, one has

$$
0 \leq d(t)<\varepsilon+\int_{0}^{t} y^{T}(\tau) u(\tau) d \tau ; \quad \forall t \in \mathbf{R}_{0+}
$$

which is coherent with a positive, negative, or null energetic interchange on $[0, t]$ with the environment satisfying $\int_{0}^{t} y^{T}(\tau) u(\tau) d \tau \geq-\varepsilon ; \forall t \in \mathbf{R}_{0+}$; and, in particular, with null such an energetic interchange, the dissipation function satisfies $0 \leq d(t)<\varepsilon ; \forall t \in \mathbf{R}_{0+}$ if $\varepsilon \in \mathbf{R}_{+}$.

Note that (38) implies that the passive system is also stable since $V(t) \leq V(0)<+\infty ; \forall t \in \mathbf{R}_{0+}$ for any finite state initial conditions. The so-called conservative system is described by the subcase of conditions (38) under the subsequent particular constraints which imply a constant storage energy defined for the given initial conditions and zero interchanged energy with the environment for any given time interval:

$$
d(t)=\int_{0}^{t} y^{T}(\tau) u(\tau) d \tau=V(0)-V(t)=0
$$

$\forall t \in \mathbf{R}_{0+}$.

Case 2 (nonpassive system in $\left[0, t_{i}\right]$ ). One has from $(36)$ that

$$
\begin{aligned}
d\left(t_{i}\right) & =\int_{0}^{t_{i}} y^{T}(\tau) u(\tau) d \tau+V(0)-V(t) \\
& <\min \left(V(0)-\left(\varepsilon_{t_{i}}+V\left(t_{i}\right)\right), 0\right) \leq 0
\end{aligned}
$$


and $\lim _{t \rightarrow \infty} \int_{0}^{t} y^{T}(\tau) u(\tau) d \tau=-\infty$ which happens if either

(1)

$$
\begin{gathered}
\left(\left[V\left(t_{0}\right)-\varepsilon_{t_{i}}-V\left(t_{i}\right) \geq 0\right] \Longleftrightarrow\right. \\
\left.\left[V\left(t_{i}\right) \leq V\left(t_{0}\right)-\varepsilon_{t}\right]\right) \Longrightarrow \\
{\left[\left(\varepsilon_{t}=0\right) \wedge\left(V\left(t_{i}\right) \leq V\left(t_{0}\right)\right)\right],}
\end{gathered}
$$

the system being stable since $V\left(t_{i}\right) \leq V(0)$ and $0>d_{t_{i}} \geq$ $\int_{0}^{t_{i}} y^{T}(\tau) u(\tau) d \tau$ which is coherent with a (negative) energetic supply on $\left[0, t_{i}\right]$ given to the environment, or if

(2)

$$
\begin{gathered}
{\left[0>V\left(t_{0}\right)-\varepsilon_{t_{i}}-V\left(t_{i}\right)\right] \Longleftrightarrow} \\
{\left[V\left(t_{i}\right)>V\left(t_{0}\right)-\varepsilon_{t}\right] .}
\end{gathered}
$$

Then, one has

$$
d\left(t_{i}\right)<\varepsilon_{t_{i}}+\int_{0}^{t_{i}} y^{T}(\tau) u(\tau) d \tau \leq 0
$$

the system being either stable if $V\left(t_{0}\right) \geq V\left(t_{i}\right)>V\left(t_{0}\right)-\varepsilon_{t_{i}}$ or unstable if $V\left(t_{i}\right)>V\left(t_{0}\right)>V\left(t_{0}\right)-\varepsilon$ and both situations are coherent with an energetic supply given to the environment on $\left[t_{0}, t\right]$ satisfying the constraint $\int_{0}^{t_{i}} y^{T}(\tau) u(\tau) d \tau<-\varepsilon_{t_{i}}<0$.

(3) Note that the situations (1) and (2) can coexist within the same interval $\left[t_{0}, t\right]$ for distinct disjoint time subintervals of nonzero measure if the control input is piecewise continuous and also if it is impulsive with a finite residence time interval in-between any two consecutive impulses. Note that a large amplitude control impulse can temporarily unstabilize a stable system or that a switched dynamic system can have switches between stable and unstable parameterizations for certain switching laws.

\section{Passivity and Switching}

Now consider a dynamic system subject to a switching law $\sigma_{s}$ : $\mathbf{R}_{0+} \rightarrow C$ with $q$ finite or infinity eventual parameterizations $C=P \cup \mathrm{NP}$, where the $p(\leq q)$ configurations (or parameterizations) $P=\left\{P_{i}: i \in \bar{p}\right\}$ are passive and the $(q-p)$ remaining ones $\mathrm{NP}=\left\{\mathrm{NP}_{i}: i \in \overline{q-p}\right\}$ are nonpassive, where $P$ is empty if $p=0$ and NP is empty if $q=p$. The switching law $\sigma_{s}: \mathbf{R}_{0+} \rightarrow C$ is defined by

$$
\begin{aligned}
& \sigma_{s}(t)=\sigma_{s}\left(t_{i}^{+}\right)=C\left(t_{i}\right)(\in C) ; \\
& \forall t \in\left[t_{i}, t_{i+1}\right), \forall t_{i} \in S W,
\end{aligned}
$$

where $t_{i}$ is an abbreviate notation for $t_{i}^{+}$versus the explicit notation for the left limit $t_{i}^{-}$with

$$
\begin{gathered}
\mathrm{SW}=\left\{t_{i} \in \mathbf{R}_{0+}: \sigma_{s}\left(t_{i}^{-}\right) \neq \sigma_{s}\left(t_{i}\right), t_{i+1}-t_{i} \geq T\right. \\
\left.>0, t_{0}=0, \quad i \in S \subseteq \mathbf{Z}_{0+}\right\}
\end{gathered}
$$

being the set of switching time instants, which can be either of infinity (denumerable) cardinal $\chi_{0}$ if the switching action never ends or finite if there is a finite final switching time. The minimum time interval in-between any two consecutive switching time instants $T$ is the minimum residence time at the active configuration. In summary, $\operatorname{card}(\mathrm{SW}) \leq \chi_{0}$ and

$$
\begin{aligned}
0 & \leq \min (\operatorname{card}(\mathrm{NP}), \operatorname{card}(P)) \\
& \leq \min (\operatorname{card}(\mathrm{NP}), \operatorname{card}(P)) \leq \operatorname{card}(C) \leq \chi_{0} .
\end{aligned}
$$

If $q<\infty$, it is possible to describe each configuration by positive integer numbers by assigning $P_{i} \rightarrow i$ and NP $\mathrm{NP}_{j} \rightarrow j-p$ for $i \in \bar{p}$ and $j \in \bar{q} \backslash \bar{p}$. Thus, the piecewise constant primary switching law $\sigma_{s}: \mathbf{R}_{0+} \rightarrow C$ is equivalently described in a simpler way by the piecewise constant switching law $\sigma$ : $\mathbf{R}_{0+} \times \mathrm{SW} \rightarrow\{1,2, \ldots, p, p+1, \ldots, q\}$ such that $\sigma(t)=\sigma\left(t_{i}\right)=$ $\ell(\in \bar{q})$ if $\sigma_{s}\left(t_{i}\right)=C_{\ell}$ if $\ell \leq p$ or $\sigma_{s}\left(t_{i}\right)=C_{\ell+i}$ if $p<\ell \leq q$. Note that

$$
\begin{array}{r}
\int_{t_{i}}^{t} y^{T}(\tau) u(\tau) d \tau \geq-\varepsilon\left(t_{i}\right)-\int_{0}^{t_{i}} y^{T}(\tau) \\
u(\tau) d \tau \\
\forall t \in\left[t_{i}, t_{i+1}\right)
\end{array}
$$

if $\sigma\left(t_{i}\right) \leq p$, that is, if the active configuration on the time interval $\left[t_{i}, t_{i+1}\right), t_{i}, t_{i+1} \in \mathrm{SW}$ is passive, where $\varepsilon\left(t_{i}\right) \in \Sigma_{P}=$ $\left\{\varepsilon_{i}: i \in \bar{p}\right\}$ and $0 \leq \varepsilon_{i} \leq \varepsilon ; \forall i \in \bar{p}$, with

$$
\begin{aligned}
& \int_{t_{i}}^{t} y^{T}(\tau) u(\tau) d \tau= \int_{0}^{t} y^{T}(\tau) u(\tau) d \tau \\
&-\int_{0}^{t_{i}} y^{T}(\tau) u(\tau) d \tau \\
& \geq-\varepsilon-\int_{0}^{t_{i}} y^{T}(\tau) u(\tau) d \tau ; \\
& \forall t \in\left[t_{i}, t_{i+1}\right), \forall t_{i}, t_{i+1} \in \mathrm{SW} .
\end{aligned}
$$

If $p<\sigma\left(t_{i}\right) \leq q$, that is, if the active configuration on the time interval $\left[t_{i}, t_{i+1}\right), t_{i}, t_{i+1} \in \mathrm{SW}$ is nonpassive, where $\varepsilon\left(t_{i}\right) \in$ $\Sigma_{\mathrm{NP}}=\left\{\varepsilon_{i}: i \in \bar{p}\right\}$ and $\varepsilon_{i} \geq \varepsilon^{\prime}>0 ; \forall i \in \bar{q} \backslash \bar{p}$.

Note that the whole set of switching time instants SW is

$$
\begin{aligned}
\mathrm{SW} & =\bigcup_{k \in S\left(\subseteq \mathbf{Z}_{0+}\right)} \mathrm{SW}(k)=\bigcup_{k \in S}\left(\mathrm{SW}_{P}(k) \cup \mathrm{SW}_{\mathrm{NP}}(k)\right) \\
& =\mathrm{SW}_{P}(k) \cup \mathrm{SW}_{\mathrm{NP}}(k) \cup \mathrm{SW}_{\mathrm{NP}},
\end{aligned}
$$

where $\mathrm{SW}(k)=\mathrm{SW}_{P}(k) \cup \mathrm{SW}_{\mathrm{NP}}(k), \mathrm{SW}_{P}(k) \subseteq \mathrm{SW}_{P}$ and $\mathrm{SW}_{\mathrm{NP}}(k) \subseteq \mathrm{SW}_{\mathrm{NP}} ; \forall k \in \mathbf{Z}_{0+}$ are, respectively, the whole set of time instants $\left\{t_{i}: i \in \bar{k} \cup\{0\}\right\} \subseteq \mathrm{SW}$ until $t_{k} \in \mathrm{SW}$ for all $k \in \mathbf{Z}_{0+}$ and its disjoint subsets associated with the active passive and nonpassive configurations which occurred in the interval $\left[0, t_{k}\right)$. Note that a system configuration is passive if $E(0, t)=\int_{0}^{t} y^{T}(\tau) u(\tau) d \tau=-\varepsilon+\widetilde{\mathcal{E}}(t) \geq-\varepsilon ; \forall t \in \mathbf{R}_{0+}$ for some real function $\widetilde{\varepsilon}: \mathbf{R}_{0+} \rightarrow \mathbf{R}_{0+}$ and some $\varepsilon \in \mathbf{R}_{0+}$. 
Assumptions 13. Under switching, a system configuration is assumed passive if for some $\varepsilon \in \mathbf{R}_{0+}$ :

(1)

$$
\begin{gathered}
E\left(t_{i}, \theta\right)=\int_{t_{i}}^{t_{i}+\theta} y^{T}(\tau) u(\tau) d \tau=-\varepsilon+\widetilde{\varepsilon}\left(t_{i}, \theta\right), \\
\theta \geq 0 ; t_{i} \in \mathrm{SW}_{P}, \\
E\left(t_{i}, \theta\right)=\int_{t_{i}}^{t_{i}+\theta} y^{T}(\tau) u(\tau) d \tau=-\varepsilon+\widetilde{\varepsilon}\left(t_{i}, \theta\right) \\
=-\varepsilon+\left(\theta-T_{\min }\right) \delta_{i}(\theta) \geq-\varepsilon ; \\
\quad \theta \geq T_{\min }, t_{i} \in \mathrm{SW}_{P}, \\
E\left(t_{i}, T_{i}\right)=\int_{t_{i}}^{t_{i+1}} y^{T}(\tau) u(\tau) d \tau=-\varepsilon+\widetilde{\varepsilon}\left(t_{i}, T_{i}\right) \\
=-\varepsilon+\left(T_{i}-T_{\min }\right) \delta_{i}\left(T_{i}\right) \geq-\varepsilon ; \\
T_{i} \geq T_{\min } ; t_{i} \in \mathrm{SW}_{P}
\end{gathered}
$$

with $\widetilde{\mathcal{\varepsilon}}\left(t_{i}, \theta\right) \in \mathbf{R} ; \forall \theta \in \mathbf{R}_{0+}$ with $\widetilde{\mathcal{E}}\left(t_{i}, 0\right) \in \mathbf{R}_{0+}$ given by $\widetilde{\mathcal{E}}\left(t_{i}, \theta\right)=\left(\theta-T_{\text {min }}\right) \delta_{i}(\theta) \geq 0 ; \theta \geq T_{\text {min }}$ for some function $\delta_{i}(\theta) \in[0, \infty) ; \forall t_{i} \in \mathrm{SW}_{P}$.

(2) A system configuration of the switched law is nonpassive if for some $\varepsilon \in \mathbf{R}_{0+}$

$$
\begin{gathered}
E\left(t_{i}, \theta\right)=\int_{t_{i}}^{t_{i}+\theta} y^{T}(\tau) u(\tau) d \tau=-\varepsilon-\left|\widetilde{\varepsilon}\left(t_{i}, \theta\right)\right|, \\
\theta \geq 0 ; t_{i} \in \mathrm{SW}_{P}, \\
E\left(t_{i}, \theta\right)=\int_{t_{i}}^{t_{i}+\theta} y^{T}(\tau) u(\tau) d \tau=-\varepsilon-\left|\widetilde{\varepsilon}\left(t_{i}, \theta\right)\right| \\
=-\varepsilon-\left(\theta-T_{\min }\right) \delta_{i}(\theta)-\varepsilon_{0 i}<-\varepsilon ; \\
\theta \geq T_{\min }, t_{i} \in \mathrm{SW}_{\mathrm{NP}}, \\
E\left(t_{i}, T_{i}\right)=\int_{t_{i}}^{t_{i+1}} y^{T}(\tau) u(\tau) d \tau=-\varepsilon-\left|\widetilde{\varepsilon}\left(t_{i}, T_{i}\right)\right| \\
=-\varepsilon-\left(T_{i}-T_{\min }\right) \delta_{i}\left(T_{i}\right)-\varepsilon_{0 i}<-\varepsilon ; \\
T_{i} \geq T_{\min } ; t_{i} \in \mathrm{SW}_{\mathrm{NP}}
\end{gathered}
$$

with $\widetilde{\mathcal{\varepsilon}}\left(t_{i}, \theta\right) \in \mathbf{R} ; \forall \theta \in \mathbf{R}_{0+}$ with $\widetilde{\mathcal{\varepsilon}}(0,0) \in \mathbf{R}_{0+}$ (if $t_{0}=0 \in$ $\left.\mathrm{SW}_{P}\right)$ given by $\widetilde{\varepsilon}\left(t_{i}, \theta\right)=\left(\theta-T_{\min }\right) \delta_{i}(\theta) \geq 0 ; \theta \geq T_{\min }$ for some constant $\varepsilon_{0 i} \in \mathbf{R}_{+}$and some function $\delta_{i}(\theta)$ with $\delta_{i}(t) \rightarrow-\infty$ as $t \rightarrow \infty ; \forall t_{i} \in \mathrm{SW}_{\mathrm{NP}}$.

The fact that $\varepsilon$ is common for all configurations is made with no loss in generality. If there is a set of such constants for the configurations, it would suffice to take the maximum of all of them as a common $\varepsilon$. The same value of $\varepsilon$ is valid by reversing the inequality for nonpassive configuration since there are extra additive thresholds $\varepsilon_{0(\cdot)}$ to modulate possible discrepancies of the necessary constants for distinct nonpassive configurations. The intuitive physical interpretation of Assumptions 13 is as follows if $t_{0}=0 \in \mathrm{SW}_{P}, E(0, t) \geq-\varepsilon$; $\forall t \in \mathbf{R}_{0+}$ as it follows for standard unswitched passivity. However, for $t_{i}(>0) \in \mathrm{SW}_{P}$ and small enough time $\theta \in$ $\left[0, T_{\min }\right)$, it can happen that $E\left(t_{i}, \theta\right)<-\varepsilon$ even if $t_{i-1} \in$ $\mathrm{SW}_{P}$ because of the switching action and the possible change with jump of value at the switching time instant of storage function from the previous active configuration to the current one. For $\theta \geq T_{\min }$, the passivity property $E\left(t_{i}, \theta\right) \geq-\varepsilon$; $\forall t \in \mathbf{R}_{0+}$ is recovered. For large enough $\theta$, it is assumed that $\lim \sup _{\theta \rightarrow \infty} \delta_{i}(t) \leq \delta_{i}^{*} \in[0, \infty)$.

The following result holds.

Theorem 14. If Assumptions 13 hold a switched system is passive for all time under a switching law $\sigma: \mathbf{R}_{0+} \times \mathrm{SW} \rightarrow$ $\{1,2, \ldots, p, p+1, \ldots, q\}$, with at least one configuration being active, if

(1) the first active configuration of the switching law on $\left[t_{0}=\right.$ $\left.0, t_{1}\right) \in \mathrm{SW}_{P}$;

(2) the switching law does not involve two consecutive active configurations being nonpassive;

(3) each active passive configuration respects a minimum residence time, quantified in the proof, which can exceed the minimum common residence time given by Assumptions 13.

Proof. The system is passive if $\int_{0}^{t} y^{T}(\tau) u(\tau) d \tau=-\varepsilon+\widetilde{\varepsilon}(t) \geq$ $-\varepsilon ; \forall t \in \mathbf{R}_{0+}$ for some real function $\widetilde{\varepsilon}: \mathbf{R}_{0+} \rightarrow \mathbf{R}_{0+}$ and some $\varepsilon \in \mathbf{R}_{0+}$. It turns out that a necessary condition for switched passivity is that the first switched configuration be passive (otherwise, the passivity condition fails for $t \in\left(0, t_{1}\right)$ ). Define the active switching passivity binary indicator function $\rho(t)=$ $1 ; \forall t \in\left[t_{i}, t_{i+1}\right), \forall t_{i} \in \mathrm{SW}_{P}, \rho(t)=0 ; \forall t \in\left[t_{i}, t_{i+1}\right), \forall t_{i} \in$ $\mathrm{SW}_{\mathrm{NP}}$. Direct calculations for any $t \in\left[t_{k}, t_{k+1}\right) ; \forall t_{k} \in \mathrm{SW}$ on the input-output energy yield

$$
\begin{gathered}
E(0, t)=\int_{0}^{t} y^{T}(\tau) u(\tau) d \tau=\sum_{i=0}^{k-1} \int_{t_{i}}^{t_{i+1}} y^{T}(\tau) u(\tau) d \tau \\
+\int_{t_{k+1}}^{t} y^{T}(\tau) u(\tau) d \tau=\sum_{t_{i} \in \mathrm{SW}_{P}(k)} \int_{t_{i}}^{t_{i+1}} y^{T}(\tau) \\
\cdot u(\tau) d \tau+\sum_{t_{i} \in S \mathrm{~W}_{\mathrm{NP}}(k)} \int_{t_{i}}^{t_{i+1}} y^{T}(\tau) u(\tau) d \tau \\
+\int_{t_{k+1}}^{t} y^{T}(\tau) u(\tau) d \tau=\sum_{t_{i} \in \mathrm{SW}_{P}(k)}\left(-\varepsilon+\left(T_{i}\right.\right. \\
\left.-T_{\mathrm{min})} \delta_{i}\left(T_{i}\right)\right)-\sum_{t_{i} \in \mathrm{SW}}\left(\varepsilon+\left(T_{\mathrm{NP}}(k)\right.\right. \\
\left.+\varepsilon_{0 i}\right)+\int_{t_{k+1}}^{t} y^{T}(\tau) u(\tau) d \tau=-\varepsilon c_{P}(k+1) \\
+\sum_{t_{i} \in \mathrm{SW}_{P}(k)}\left(T_{i}-T_{\min }\right) \delta_{i}\left(T_{i}\right)-\varepsilon c_{\mathrm{NP}}(k) \\
+\sum_{t_{i} \in \mathrm{SW}_{\mathrm{NP}}(k)}\left[\left(T_{i}-T_{\min }\right) \delta_{i}\left(T_{i}\right)+\varepsilon_{0 i}\right]+\int_{t_{k+1}}^{t} y^{T}(\tau)
\end{gathered}
$$




$$
\begin{aligned}
& \cdot u(\tau) d \tau=-(k+1) \varepsilon \\
& +\left(\sum _ { t _ { i } \in \mathrm { SW } ( k + 1 ) } \left\{\rho\left(t_{i}\right)\left(T_{i}-T_{\min }\right) \delta_{i}\left(T_{i}\right)\right.\right. \\
& \left.\left.-\left(1-\rho\left(t_{i}\right)\right)\left[\left(T_{i}-T_{\min }\right) \delta_{i}\left(T_{i}\right)+\varepsilon_{0 i}\right]\right\}\right) \\
& +\int_{t_{k+1}}^{t} y^{T}(\tau) u(\tau) d \tau=-k \varepsilon \\
& +\left(\sum _ { t _ { i } \in \mathrm { SW } ( k ) } \left\{\rho\left(t_{i}\right)\left(T_{i}-T_{\min }\right) \delta_{i}\left(T_{i}\right)\right.\right. \\
& \left.\left.-\left(1-\rho\left(t_{i}\right)\right)\left[\left(T_{i}-T_{\min }\right) \delta_{i}\left(T_{i}\right)+\varepsilon_{0 i}\right]\right\}\right)+\rho\left(t_{k}\right) \\
& \cdot\left(T_{k}-T_{\min }\right) \delta_{k}\left(T_{k}\right)-\left(1-\rho\left(t_{k}\right)\right)\left[\left(T_{k}-T_{\min }\right)\right. \\
& \left.\cdot \delta_{k}\left(T_{i k}\right)+\varepsilon_{0 k}\right]+\int_{t_{k+1}}^{t} y^{T}(\tau) u(\tau) d \tau=E(0, t \\
& \left.-T_{k}\right)-\varepsilon+\rho\left(t_{k}\right)\left(T_{k}-T_{\min }\right) \delta_{k}\left(T_{k}\right)-(1 \\
& \left.-\rho\left(t_{k}\right)\right)\left[\left(T_{k}-T_{\min }\right) \delta_{k}\left(T_{i k}\right)+\varepsilon_{0 k}\right]+\int_{t_{k+1}}^{t} y^{T}(\tau) \\
& \cdot u(\tau) d \tau \geq-2 \varepsilon+\rho\left(t_{k}\right)\left(T_{k}-T_{\min }\right) \delta_{k}\left(T_{k}\right) \\
& -\left(1-\rho\left(t_{k}\right)\right)\left[\left(T_{k}-T_{\min }\right) \delta_{k}\left(T_{i k}\right)+\varepsilon_{0 k}\right] \\
& +\int_{t_{k+1}}^{t} y^{T}(\tau) u(\tau) d \tau \geq-\varepsilon ; \quad \forall t \in\left[t_{k}, t_{k+1}\right)
\end{aligned}
$$

holds via complete induction if $t_{0}(=0) \in \mathrm{SW}_{P}$ (i.e., the first configuration of the switched disposal is passive) provided that $E\left(0, t-T_{k}\right) \geq-\varepsilon$ for any $t \in \mathbf{R}_{0+}\left(t-T_{k}\right) \in\left[t_{k-1}, t_{k 1}\right)$ if the subsequent constraint holds:

$$
\begin{aligned}
\rho\left(t_{k}\right) & \left(T_{k}-T_{\min }\right) \delta_{k}\left(T_{k}\right) \\
\geq & \varepsilon+\left(1-\rho\left(t_{k}\right)\right)\left[\left(T_{k}-T_{\min }\right) \delta_{k}\left(T_{i k}\right)+\varepsilon_{0 k}\right] \\
& -\int_{t_{k+1}}^{t} y^{T}(\tau) u(\tau) d \tau .
\end{aligned}
$$

The following cases can occur:

Case 1. $t_{k}, t_{k+1} \in \mathrm{SW}_{P}$. Thus, $\rho\left(t_{k}\right)=1$ and

$$
\begin{aligned}
\left(T_{k}-T_{\min }\right) \delta_{k}\left(T_{k}\right) \geq & 2 \varepsilon \\
& -\left(T_{k+1}-T_{\min }\right) \delta_{k+1}\left(t-t_{k+1}\right)
\end{aligned}
$$

which is guaranteed if

$$
T_{k+1} \geq T_{\min }+\min \left(\frac{2 \varepsilon-\left(T_{k}-T_{\min }\right) \delta_{k}\left(T_{k}\right)}{\inf _{t \in\left[t_{k}, t_{k+1}\right)} \delta_{k+1}\left(t-t_{k+1}\right)}, 0\right)
$$

or if

$$
\begin{aligned}
& T_{k+1} \geq T_{\min } \\
& +\min \left(\frac{\varepsilon-\inf _{t \in\left[t_{k}, t_{k+1}\right)} E\left(0, t-t_{k}\right)-\left(T_{k}-T_{\min }\right) \delta_{k}\left(T_{k}\right)}{\inf _{t \in\left[t_{k}, t_{k+1}\right)} \delta_{k+1}\left(t-t_{k+1}\right)},\right. \\
& \quad 0) .
\end{aligned}
$$

Case 2. $t_{k}, t_{k+1} \in \mathrm{SW}_{\mathrm{NP}}$. Thus, $\rho\left(t_{k}\right)=0$ and

$$
\begin{gathered}
0 \geq 2 \varepsilon+\left[\left(T_{k}-T_{\min }\right) \delta_{k}\left(T_{i k}\right)+\varepsilon_{0 k}\right]+\varepsilon_{0, k+1} \\
+\left(t-t_{k+1}-T_{\min }\right) \delta_{k+1}\left(t-t_{k+1}\right) .
\end{gathered}
$$

Case 3. $t_{k} \in \mathrm{SW}_{\mathrm{NP}}, t_{k+1} \in \mathrm{SW}_{P}$. Thus, $\rho\left(t_{k}\right)=0$ and

$$
\begin{aligned}
0 \geq & 2 \varepsilon+\left[\left(T_{k}-T_{\min }\right) \delta_{k}\left(T_{i k}\right)+\varepsilon_{0 k}\right] \\
& -\left(t-t_{k+1}-T_{\min }\right) \delta_{k+1}\left(t-t_{k+1}\right)
\end{aligned}
$$

yields closely to Case 1:

$$
\begin{aligned}
T_{k+1} \geq & T_{\min } \\
& +\min \left(\frac{2 \varepsilon+\varepsilon_{0, k+1}-\left(T_{k}-T_{\min }\right) \delta_{k}\left(T_{k}\right)}{\inf _{t \in\left[t_{k}, t_{k+1}\right.} \delta_{k+1}\left(t-t_{k+1}\right)}, 0\right) .
\end{aligned}
$$

\section{Examples}

Example 15. Consider the following damped linear system:

$$
\begin{aligned}
& \dot{x}(t)=\frac{p(t)}{m} ; \\
& \dot{p}(t)=m \ddot{x}(t)=F(t)-k x(t)-\lambda \dot{x}(t) ; \\
& x(0)=x_{0}, \\
& \dot{x}(0)=\dot{x}_{0}
\end{aligned}
$$

with $x(t)$ being the position, $y(t)=\dot{x}(t)$ being the velocity, defined to be the system output, $u(t)=F(t)$ being the external force, defined to be the control action, and $k$ and $\lambda$ being the spring constant and the viscous coefficient, respectively. Define the state vector as $z(t)=(x(t), \dot{x}(t))^{T}$ and let $V(z(t))=$ $(1 / 2) m \dot{x}^{2}(t)+(1 / 2) k x^{2}(t)=V_{\text {kin }}(\dot{x}(t))+V_{\text {pot }}(x(t))$ be the storage function defined to be the kinetic energy $V_{\text {kin }}(\dot{x}(t))$ plus the potential energy $V_{\text {pot }}(x(t))$. The input-output energy at time $t \in \mathbf{R}_{0+}$ is

$$
\begin{aligned}
E(t) & =\int_{0}^{t} y(\tau) u(\tau) d \tau=\int_{0}^{t} \dot{x}(\tau) F(\tau) d \tau \\
& =\int_{0}^{t} \dot{x}(\tau)[m \ddot{x}(\tau)+k x(\tau)+\lambda \dot{x}(\tau)] d \tau \\
& =V(z(t))-V(z(0))+d(t)
\end{aligned}
$$


where the dissipation function at time $t$ is $d(t)=$ $\lambda \int_{0}^{t} \dot{x}^{2}(\tau) d \tau \geq 0 ; \forall t \in \mathbf{R}_{0+}$. If $\lambda=0, x(t)=x_{0}, F(t)=$ $0, \dot{x}(t)=\dot{x}_{0} ; \forall t \in \mathbf{R}_{0+}$ then $V(z(t))=V\left(z_{0}\right)=(1 / 2) m \dot{x}_{0}^{2}+$ $(1 / 2) k x_{0}^{2}, E(t)=d(t)=0 ; \forall t \in \mathbf{R}_{0+}$ and the system is a conservative system (a particular case of dissipative system) with constant stored energy, zero dissipation for all time, and zero input-output energy for all time which implies also that the system is globally stable (i.e., stable for any initial conditions). If there exists some real constant $\gamma$ such that $V(z(t))-V(z(0))+d(t) \geq-\gamma^{2} ; \forall t \in \mathbf{R}_{0+}$, that is,

$$
\begin{aligned}
+\infty>d(t)+\gamma^{2} \geq V(z(0))-V(z(t))>-\infty & \\
& \forall t \in \mathbf{R}_{0+}
\end{aligned}
$$

then the system is passive. In the case that the passive system is also globally asymptotically stable, $z(t) \rightarrow 0, V(z(t)) \rightarrow$ $V(0)=0$ as $t \rightarrow \infty$ and $0 \leq d(t) \leq d\left(t^{\prime}\right)<+\infty$ for all $t^{\prime} \in[t, \infty) ; \forall t \in \mathbf{R}_{0+}$ and $d(\infty)=\int_{0}^{\infty} \dot{x}^{2}(t) d t<+\infty$. Sufficient conditions are obtained by giving conditions on the stored energy to be a Lyapunov function for global asymptotic stability as follows:

$$
\begin{aligned}
\dot{V}(z(t))= & \frac{d}{d t\left((1 / 2) m \dot{x}^{2}(t)+(1 / 2) k x^{2}(t)\right)} \\
= & m \dot{x}(t) \ddot{x}(t)+k x(t) \dot{x}(t) \\
= & m \dot{x}(t)(F(t)-k x(t)-\lambda \dot{x}(t)) \\
& +k x(t) \dot{x}(t) \leq 0 ; \quad \forall t \in \mathbf{R}_{0+}
\end{aligned}
$$

if the external force is generated via feedback as follows:

$$
\begin{aligned}
F(t)= & \frac{m-1}{m} k x(t) \dot{x}(t)+\lambda \dot{x}^{2}(t)-\zeta(x(t), \dot{x}(t), t) \\
= & \frac{1}{m}\left[(m-1) \dot{V}_{\mathrm{pot}}(t)+\lambda V_{\mathrm{kin}}(t)\right] \\
& -\zeta(x(t), \dot{x}(t), t) ; \quad \forall t \in \mathbf{R}_{0+},
\end{aligned}
$$

where $\zeta:\left(\mathbf{R} \times \mathbf{R}_{0+}\right) \times\left(\mathbf{R} \times \mathbf{R}_{0+}\right) \times \mathbf{R}_{0+} \rightarrow \mathbf{R}_{0+}$ is a design function so that $\zeta(x(t), \dot{x}(t), t)=0$ if and only if $x(t)=0$. Thus, $V(z(t)) \leq V\left(z_{0}\right) ; \forall t \in \mathbf{R}_{0+}$ and $V(z(t)) \rightarrow 0$ as $t \rightarrow \infty$ and the passive system is globally asymptotically stable.

In the case that the damping device has a nonlinear cubic effect (forced Duffing equation), the motion and Lyapunov function time-derivative satisfy

$$
\begin{aligned}
& m \ddot{x}(t)= F(t)-k x(t)-\beta x^{3}(t)-\lambda \dot{x}(t) ; \\
& \forall t \in \mathbf{R}_{0+}, \\
& \dot{V}(z(t))= m \dot{x}(t)\left(F(t)-k x(t)-\beta x^{3}(t)-\lambda \dot{x}(t)\right) \\
&+k x(t) \dot{x}(t) \leq 0 ; \quad \forall t \in \mathbf{R}_{0+}
\end{aligned}
$$

if

$$
\begin{aligned}
F(t)= & \frac{m-1}{m} k\left[1+\frac{\beta}{k} x(t)\right](x(t)+\lambda \dot{x}(t)) \dot{x}(t) \\
& -\zeta(x(t), \dot{x}(t), t) ; \quad \forall t \in \mathbf{R}_{0+} .
\end{aligned}
$$

If $F(t)=\sigma(t) x(t)+\sigma_{d} \dot{x}(t)$ then $\dot{V}(z(t)) \leq 0$ and global stability holds if $\sigma_{d}=\lambda$ and $\sigma(t)=k((m-1) / m)-\beta x^{2}(t)$; $\forall t \in \mathbf{R}_{0+}$ since the exact output linearization dynamics is achieved under such a control.

Example 16. Consider a $n$th order SISO time-invariant linear system whose transfer function is $\widehat{G}(s)=c^{T}\left(s I_{n}-A\right)^{-1} b+d$, where $\left\{A, b, c^{T}, d\right\}$ is a state-space realization of $\widehat{G}(s)$, and the state $n$-vector has initial conditions $x(0)=x_{0}$. The output is given by a superposition of the unforced (i.e., zero -input) and forced (i.e., zero state) solutions $y_{\text {unf }}(t)$ and $y_{f}(t)$ as

$$
\begin{aligned}
y(t) & =y_{\mathrm{unf}}(t)+y_{f}(t) \\
& =c^{T} e^{A t}\left(x_{0}+\int_{0}^{t} e^{-A \tau} b u(\tau) d \tau\right)+d u(t),
\end{aligned}
$$

where

$$
y_{f}(t)=y(t)-y_{\mathrm{unf}}(t)=\int_{0}^{t} c^{T} e^{A(t-\tau)} b u(\tau)+d u(t)
$$

Then, by using Parseval's theorem, we can equalize the inputoutput energy evaluation in the time domain to its evaluation in the frequency domain as follows provided that the Fourier transforms of the input and output signals exist:

$$
\begin{aligned}
E_{f}(t) & =\int_{0}^{t} y_{f}(\tau) u(\tau) d \tau=\int_{0}^{\infty} y_{f}(\tau) u_{t}(\tau) d \tau \\
& =\frac{1}{2 \pi} \int_{0}^{\infty} \widehat{y}_{f}(\mathbf{i} \omega) \widehat{u}_{t}(-\mathbf{i} \omega) d \omega \\
& =\frac{1}{2 \pi} \int_{0}^{\infty} \widehat{y}_{t}(\mathbf{i} \omega) \widehat{u}_{t}(-\mathbf{i} \omega) d \omega \\
& =\frac{1}{2 \pi} \int_{-\infty}^{\infty} \widehat{G}(\mathbf{i} \omega) \widehat{u}_{t}(\mathbf{i} \omega) \widehat{u}_{t}(-\mathbf{i} \omega) d \omega \\
& =\frac{1}{2 \pi} \int_{-\infty}^{\infty} \widehat{G}(\mathbf{i} \omega)\left|\widehat{u}_{t}(\mathbf{i} \omega)\right|^{2} d \omega \\
& \geq \frac{1}{2 \pi} \min _{\omega \in \mathbf{R}_{0+}} \widehat{G}(\mathbf{i} \omega) \int_{-\infty}^{\infty}\left|\widehat{u}_{t}(\mathbf{i} \omega)\right|^{2} d \omega \\
& =\frac{1}{2 \pi} \min _{\omega \in \mathbf{R}_{0+}} \widehat{G}(\mathbf{i} \omega) \int_{-\infty}^{\infty}\left|\widehat{u}_{t}(\mathbf{i} \omega)\right|^{2} d \omega \\
& =\min _{\omega \in \mathbf{R}_{0+}} \widehat{G}(\mathbf{i} \omega) \int_{0}^{\infty}\left|u_{t}(\tau)\right|^{2} d \tau \\
& =\min _{\omega \in \mathbf{R}_{0+}} \widehat{G}(\mathbf{i} \omega) \int_{0}^{t} u^{2}(\tau) d \tau, \forall t \in \mathbf{R}_{0+}
\end{aligned}
$$

since $\operatorname{Im} \widehat{G}(\mathbf{i} \omega)=-\operatorname{Im} \widehat{G}(\mathbf{i} \omega)$ for any hodograph $\widehat{G}(\mathbf{i} \omega) ; \forall \omega \epsilon$ $\mathbf{R}_{0+}$. Assume that $\widehat{G} \in\{\mathrm{PR}\}$ so that $E_{f}(t) \geq d \int_{0}^{\infty} u^{2}(\tau) d \tau$ from (71). Assume also that the control is generated via negative output-feedback from any member $\phi \in\{\Phi\}$ of nonlinear and perhaps time-varying class of controllers $\{\Phi\}$ 
which satisfies a Popov's type inequality, namely, $u(t)=$ $-\phi(y(t), t)$, where

$$
\begin{aligned}
\int_{0}^{t} y(\tau) u(\tau) d \tau & =-\int_{0}^{t} y(\tau) \phi(y(\tau), \tau) d \tau=-E(t) \\
& \geq-\gamma(\gamma>0)
\end{aligned}
$$

so that for any given positive real $\bar{\gamma}$ such that $\bar{\gamma} \geq \gamma$ and any nonidentically zero controls, one gets

$$
\begin{gathered}
+\infty>\bar{\gamma} \geq \gamma \geq E(t)=\int_{0}^{t} y(\tau) u(\tau) d \tau \\
\geq d \int_{0}^{t} u^{2}(\tau) d \tau>0 ; \quad \forall t \in \mathbf{R}_{0+}
\end{gathered}
$$

so that for nonidentically zero controls

$$
\begin{aligned}
+\infty & >\bar{\gamma}-E_{\mathrm{unf}}(t)=\bar{\gamma}-\int_{0}^{t} y_{\mathrm{unf}}(\tau) u(\tau) d \tau \\
& \geq \gamma-\int_{0}^{t} y_{\mathrm{unf}}(\tau) u(\tau) d \tau \\
& =\gamma-\int_{0}^{t} c^{T} e^{A(t-\tau)} x_{0} u(\tau) d \tau \\
& =\gamma-\int_{0}^{t} c^{T} e^{A(t-\tau)} x_{0} u(\tau) d \tau \geq \int_{0}^{t} y_{f}(\tau) u(\tau) d \tau \\
& \geq d \int_{0}^{t} u^{2}(\tau) d \tau>0 ; \quad \forall t \in \mathbf{R}_{0+}
\end{aligned}
$$

The following result holds from (74).

Theorem 17. The following properties hold:

(i) Assume that $\widehat{G} \in\{S S P R\}$. Then, $u, y \in L_{2}$ and the system is globally asymptotically stable for any given finite initial conditions, irrespective of $\phi \in\{\Phi\}$, so that it is asymptotically hyperstable.

(ii) Assume that $\widehat{G} \in\{S P R\}$ and that a frequency filter $\widehat{F}(\mathbf{i} \omega)$ is used for the control inputs so that $\widehat{F}(\mathbf{i} \omega) \widehat{u}(\mathbf{i} \omega)=0$ for any frequency interval, if any, such that $\min \operatorname{Re} \widehat{G}(\mathbf{i} \omega)=0$ and it is of unity gain otherwise. Then, $u, y \in L_{2}$ and the system is globally asymptotically stable for any given finite initial conditions, irrespective of $\phi \in\{\Phi\}$; then it is asymptotically hyperstable for the forward loop transfer function $\widehat{G}(s) \widehat{F}(s)$.

Proof. Since $\widehat{G} \in\{\mathrm{SSPR}\}$ so that $d>0$ and $\widehat{G} \in\{\mathrm{SPR}\}$, that is, it is strongly positive real then, in addition, in $\mathbf{R} \mathbf{H}_{\infty}$ (then with all its poles in $\operatorname{Re} s<0$ ) and having zero relative degree it follows in (74) that $+\infty>\bar{\gamma}=\bar{\gamma}\left(x_{0}, u\right)>$ $\max \left(\gamma, \int_{0}^{t} c^{T} e^{A(t-\tau)} x_{0} u(\tau) d \tau\right)$ can be made and the Fourier transforms used in the Parseval's identity exist. Assume that $u(t) \rightarrow \pm \infty$ as $t \rightarrow \infty$ then, there exists $\alpha \in \mathbf{R}_{+}$such that

$$
\begin{aligned}
\gamma & \geq \limsup _{t \rightarrow \infty}\left((d+\alpha) \int_{0}^{t} u^{2}(\tau) d \tau\right) \\
& \geq \limsup _{t \rightarrow \infty}\left(d \int_{0}^{t} u^{2}(\tau) d \tau+\int_{0}^{t} y_{\text {unf }}(\tau) u(\tau) d \tau\right)
\end{aligned}
$$

since $u^{2}(t) / u(t) \rightarrow \infty$ and $e^{A t} \rightarrow 0$ as $t \rightarrow \infty$. Therefore, $u \in L_{2}$, so that $u(t) \rightarrow 0$ as $t \rightarrow \infty$ which contradicts that $u(t) \rightarrow \pm \infty$ as $t \rightarrow \infty$. It can also be concluded that $u(t)$ is bounded on any finite time interval and it can be infinity only on a set of zero measure (i.e., it can be eventually impulsive only at isolated time instants). Thus, one concludes that the input is almost everywhere bounded and it converges asymptotically to zero as time tends to infinity. Note that (71) implies that $E_{f}(t) \geq \min _{\omega \in \mathbf{R}_{0+}} \widehat{G}^{-1}(\mathbf{i} \omega) \int_{0}^{t} y^{2}(\tau) d \tau$ with $\widehat{G}^{-1} \epsilon$ $\{\mathrm{SSPR}\}$ and is realizable, since $\widehat{G} \in\{\mathrm{SSPR}\}$ so it has relative degree zero. Thus, $y \in L_{2}$ and converges asymptotically to zero for any $\phi \in\{\Phi\}$. Since $\widehat{G} \in \mathbf{R H}_{\infty}$ the system is globally asymptotically stable for any given initial conditions so that the state, control input, and output are uniformly bounded for all time and converge asymptotically to zero as time tends to infinity. Property (i) has been proved. To prove Property (ii), note that

$$
\begin{aligned}
E_{f}(t) & \geq \frac{1}{2 \pi} \min _{\omega \in \mathbf{R}_{0+}} \widehat{G}(\mathbf{i} \omega) \int_{-\infty}^{\infty}\left|\widehat{u}_{t}(\mathbf{i} \omega)\right|^{2} d \omega \\
& =\frac{1}{2 \pi} \sum_{j=1}^{r} \min _{\omega \in\left[\omega_{j}, \omega_{j+1}\right)} \widehat{G}_{j}(\mathbf{i} \omega) \int_{\omega_{j}}^{\omega_{j+1}}\left|\widehat{u}_{t j}(\mathbf{i} \omega)\right|^{2} d \omega \\
& \geq \sum_{j=1}^{r} \min _{\omega \in\left[\omega_{j}, \omega_{j+1}\right)} \widehat{G}_{j}(\mathbf{i} \omega) \int_{0}^{\infty} u_{t j}^{2}(\tau) d \tau \\
& =\sum_{j \in \vartheta(r)} d_{j} \int_{0}^{\infty} u_{t j}^{2}(\tau) d \tau=\sum_{j \in \vartheta(r)} d_{j} \int_{0}^{t} u_{j}^{2}(\tau) d \tau,
\end{aligned}
$$

where $d_{j}=\min _{\omega \in\left[\omega_{j}, \omega_{j+1}\right)} \widehat{G}_{j}(\mathbf{i} \omega) \geq 0$ since $\widehat{G} \in\{\mathrm{PR}\}$ and $\vartheta(r) \subseteq \bar{r}$ is the indicator set such that $d_{j}>0$ if and only if $j \in \vartheta(r)$. Thus, $u_{j} \in L_{2}, u_{j}$ is bounded; then $u_{j}(t) \rightarrow 0$ as $t \rightarrow$ $\infty$ since $\widehat{G} \in\{$ SPR $\}$ for all $j \in \mathcal{\vartheta}(r)$ and the total control input $u(t)=\sum_{j \in \vartheta(r)} u_{j}(t)+\sum_{j \in \bar{r} \backslash \vartheta(r)} u_{j}(t)$ is also bounded since $u_{j}$ is bounded by the filtering actions; $\forall j \in \bar{r} \backslash \vartheta(r)$. If $u_{j} \equiv 0 ; \forall j \in$ $\bar{r} \backslash \vartheta(r)$ then Property (i) holds for this class of constrained control inputs.

Example 18. Consider a $n$th order SISO time-invariant linear system described by a finite or infinite set of transfer functions $\left\{\widehat{G}_{j}(s): j \in \bar{r}\right\}$ governed by a switching law $\sigma: \bigcup_{k}\left[t_{k}, t_{k+1}\right) \rightarrow$ $\bar{r}$, with $\operatorname{card} \bar{r} \leq \chi_{0}$, which selects the active one on a time interval $\left[t_{k}, t_{k+1}\right)$ where $\mathrm{SW}=\left\{t_{k}\right\}$ is a finite or infinite set of switching time instants. Stability preservation or achievement under switched laws governing switched parameterizations has been studied in the background literature. It is of interest to extend it to positivity and passivity properties under switching laws. See, for instance, [14]. Let a time instant $t \in$ $\left(t_{k+1}, t_{k+2}\right]$. To simplify the exposition, consider the system under zero initial conditions. Then

$$
\begin{gathered}
E_{f}(t)=\sum_{j=0}^{k} \int_{t_{j}}^{t_{j+1}} y(\tau) u(\tau) d \tau+\int_{t_{k+1}}^{t} y(\tau) u(\tau) d \tau \\
=\sum_{j=0}^{k} \int_{0}^{\infty} y(\tau) u_{t_{j}, t_{j+1}}(\tau) d \tau
\end{gathered}
$$




$$
\begin{aligned}
& +\int_{0}^{\infty} y(\tau) u_{t_{k+1}, t}(\tau) d \tau \\
& \geq \frac{1}{2 \pi}\left[\sum_{j=0}^{k} \min _{\omega \in \mathbf{R}_{0+}} \widehat{G}_{\sigma\left(t_{k}\right)}(\mathbf{i} \omega) \int_{-\infty}^{\infty}\left|\widehat{u}_{t_{j}, t_{j+1}}(\mathbf{i} \omega)\right|^{2} d \omega\right. \\
& \left.+\min _{\omega \in \mathbf{R}_{0+}} \widehat{G}_{\sigma\left(t_{k}\right)}(\mathbf{i} \omega) \int_{-\infty}^{\infty}\left|\widehat{u}_{t_{k}, t}(\mathbf{i} \omega)\right|^{2} d \omega\right] \\
& =\sum_{j=0}^{k} \min _{\omega \in \mathbf{R}_{0+}} \widehat{G}_{\sigma\left(t_{k}\right)}(\mathbf{i} \omega) \int_{0}^{t} u_{t_{j}, t_{j+1}}^{2}(\tau) d \tau \\
& +\min _{\omega \in \mathbf{R}_{0+}} \widehat{G}_{\sigma\left(t_{k}\right)}(\mathbf{i} \omega) \int_{t_{k+1}}^{t} u_{t_{k+1}, t}^{2}(\tau) d \tau \\
& \geq \max \left(\zeta_{1 t}, \zeta_{2 t}\right),
\end{aligned}
$$

where a truncated interswitching input $u_{t_{a}, t_{b}}(t)=u(t)$ for $t \epsilon$ $\left[t_{a}, t_{b}\right]$ and $u_{t_{a}, t_{b}}(t)=0$ for $t \notin\left[t_{a}, t_{b}\right]$ has been defined and

$$
\begin{aligned}
& \zeta_{1 t}=\left(\sum_{j=0}^{k} \min _{\omega \in \mathbf{R}_{0+}} \widehat{G}_{\sigma\left(t_{j}\right)}(\mathbf{i} \omega)+\min _{\omega \in \mathbf{R}_{0+}} \widehat{G}_{\sigma\left(t_{k+1}\right)}(\mathbf{i} \omega)\right) \\
& \cdot \min \left(\min _{j \in \bar{k} \cup\{0\}} \int_{0}^{t} v_{j} u_{t_{j}, t_{j+1}}^{2}(\tau) d \tau,\right. \\
& \left.\int_{0}^{t} v_{k+1} u_{t_{k+1}, t}^{2}(\tau) d \tau\right)=\left(\sum_{j=0}^{k} \min _{\omega \in \mathbf{R}_{0+}} \widehat{G}_{\sigma\left(t_{j}\right)}(\mathbf{i} \omega)\right. \\
& \left.+\min _{\omega \in \mathbf{R}_{0+}} \widehat{G}_{\sigma\left(t_{k}\right)}(\mathbf{i} \omega)\right) \min \left(\min _{j \in \bar{k} \cup\{0\}} \int_{t_{j}}^{t_{j+1}} v_{j} u^{2}(\tau) d \tau,\right. \\
& \left.\int_{t_{k+1}}^{t} v_{k} u^{2}(\tau) d \tau\right) \\
& \zeta_{2 t}=\min \left(\min _{j \in \bar{k} \cup\{0\}} \min _{\omega \in \mathbf{R}_{0+}} \widehat{G}_{\sigma\left(t_{j}\right)}, \min _{\omega \in \mathbf{R}_{0+}} \widehat{G}_{\sigma\left(t_{k}\right)}(\mathbf{i} \omega)\right) \\
& \int_{0}^{\infty} u^{2}(\tau) d \tau
\end{aligned}
$$

with

$$
v_{j}= \begin{cases}1 & \text { if } \min _{\omega \in \mathbf{R}_{0+}} \widehat{G}_{\sigma\left(t_{j}\right)}(\mathbf{i} \omega)>0 \\ 0 & \text { if } \min _{\omega \in \mathbf{R}_{0+}} \widehat{G}_{\sigma\left(t_{j}\right)}(\mathbf{i} \omega)=0 \\ -1 & \text { if } \min _{\omega \in \mathbf{R}_{0+}} \widehat{G}_{\sigma\left(t_{j}\right)}(\mathbf{i} \omega)<0 .\end{cases}
$$

The following related result holds.

Theorem 19. The following properties hold:

(i) Assume that the switching law incorporates a zero state resetting action at each $t_{j} \in S W$. Then, the switched system is positive and passive for such a switching law if all the transfer functions involved by the switching law are positive real. (ii) Assume that the switching law incorporates a zero state resetting action at each $t_{j} \in S W$. Assume also that the switching law satisfies that $G_{\sigma\left(t_{0}\right)} \in\{S P R\}$ and that $G_{\sigma\left(t_{k}\right)} \in \mathbf{R H}_{\infty}$ and that, in the event that $G_{\sigma\left(t_{k}\right)} \in \mathbf{R H}_{\infty} \backslash\{P R\}$ for $k \in \mathbf{Z}_{+}$, then $G_{\sigma\left(t_{k+1}\right)} \in\{S P R\}$, and

$$
\begin{aligned}
t_{k+2} & <\inf \left(t>t_{k+1}: \int_{t_{k+1}}^{t} u^{2}(\tau) d \tau\right. \\
& \left.\leq \frac{\sum_{j=0}^{k} \min _{\omega \in \mathbf{R}_{0+}} \widehat{G}_{\sigma\left(t_{k}\right)}(\mathbf{i} \omega) \int_{t_{j}}^{t_{j+1}} u^{2}(\tau) d \tau}{\left|\max _{\omega \in \mathbf{R}_{0+}} \widehat{G}_{\sigma\left(t_{k}\right)}(\mathbf{i} \omega)\right|}\right) .
\end{aligned}
$$

Then, the switched system is strictly input passive for such a switching law.

(iii) Assume that the system is subject to a feedback control law $\phi \in\{\Phi\},(72)$, and that $\widehat{G}_{\sigma\left(t_{j}\right)} \in\{S P R\} ; \forall t_{j} \in S W$ with $\widehat{G}_{\sigma\left(t_{0}\right)} \in\{S S P R\}$ and to zero state resetting at each switching time instant. Then, $u, y \in L_{2}$ and the system is globally asymptotically stable for any given finite initial conditions, irrespective of $\phi \in\{\Phi\}$, so that it is asymptotically hyperstable.

(iv) Assume also that the constraints $\widehat{G}_{\sigma\left(t_{0}\right)} \in\{S S P R\}$ and zero state switching resetting invoked in Property (iii) but frequency filters $\widehat{F}_{\sigma\left(t_{j}\right)}(\mathbf{i} \omega)$ are used for the control inputs so that $\widehat{F}_{\sigma\left(t_{j}\right)}(\mathbf{i} \omega) \widehat{u}(\mathbf{i} \omega)=0$ for any frequency interval, if any, such that $\min \operatorname{Re} \widehat{G}_{\sigma\left(t_{j}\right)}(\mathbf{i} \omega)=0$ and it is of unity gain otherwise for $t_{j} \epsilon$ $S W$. Then, $u, y \in L_{2}$ and the system is globally asymptotically stable for any given finite initial conditions, irrespective of $\phi \in$ $\Phi$; then it is asymptotically hyperstable for the forward loop transfer function $\widehat{G}(s) \widehat{F}(s)$.

Proof. Property (i) follows from (77a), (77b), and (78) and $E(t)=E_{f}(t) \geq 0 ; \forall t \in \mathbf{R}_{0+}$ under zero state resetting at each switching time instant since

$$
\sum_{j=0}^{k} \min _{\omega \in \mathbf{R}_{0+}} \widehat{G}_{\sigma\left(t_{k}\right)}(\mathbf{i} \omega)+\min _{\omega \in \mathbf{R}_{0+}} \widehat{G}_{\sigma\left(t_{k}\right)}(\mathbf{i} \omega) \geq 0
$$

for any $k \in \mathbf{Z}_{0+}$, any $t_{k} \in \mathrm{SW}$ and any $t \in\left(t_{k}, t_{k+1}\right)$. Property (ii) by taking into account (77a) and $G_{\sigma\left(t_{0}\right)} \in\{\mathrm{SPR}\}$ which guarantees that $E_{f}(t)=E(t)>0 ; \forall t \in \mathbf{R}_{+}$and that (77a) and (77b) holds since any active stable, irrespective of it is positive or nonpositive real, transfer function is in active operation a sufficiently small time interval to guarantee that $E(t)=E_{f}(t)>0 ; \forall t \in \mathbf{R}_{+}$under zero resetting of the state conditions at any switching time instant. Properties (iii)-(iv) follow from Property (iii) and Theorem 17.

Example 20. Use the convolution expression for the zero state energy measure in Example 18 to yield

$$
\begin{aligned}
E_{f}(t) & =\int_{0}^{t}\left(\int_{0}^{\tau} G(\tau-\theta) u(\theta) d \theta\right) u(\tau) d \tau \\
= & \sum_{j=0}^{k} \sum_{\ell=0}^{k_{\tau}} \int_{t_{j}}^{t_{j+1}}\left(\int_{t_{\ell}}^{t_{\ell+1}} G_{\ell}(\tau-\theta) u_{\ell}(\theta) u_{j}(\tau) d \theta d \tau\right)
\end{aligned}
$$




$$
\begin{aligned}
& +\sum_{j=0}^{k} \int_{t_{j}}^{t_{j+1}} \int_{t_{k_{\tau}}+1}^{\tau} G_{t_{k_{\tau}}+1}(\tau-\theta) u_{t_{k_{\tau}}+1}(\theta) u_{j}(\tau) d \theta d \tau \\
& +\sum_{\ell=0}^{k_{\tau}} \int_{t_{k+1}}^{t} \int_{t_{\ell}}^{t_{\ell+1}} G_{\ell}(\tau-\theta) u_{\ell}(\theta) u_{k+1}(\tau) d \theta d \tau \\
& +\int_{t_{k+1}}^{t} \int_{t_{k_{\tau}}+1}^{\tau} G_{t_{k_{\tau}}+1}(\tau-\theta) u_{t_{k_{\tau}}+1}(\theta) u_{k+1}(\tau) d \theta d \tau .
\end{aligned}
$$

We find the following properties if the system is externally positive in the sense that for any nonnegative controls and initial conditions the output is nonnegative for all time:

(i) If all the transfer functions of the switching law have a state-space representation where the impulse response $c_{(\cdot)}^{T} e^{A_{(\cdot)} b_{(\cdot)}}+d_{(\cdot)}$ is nonnegative for all time and the controls are also everywhere nonnegative in the definition domain then the output is nonnegative for all time and the input-output energy is also nonnegative for all time provided that the initial state is zero and subject to reset to zero at each switching time instant.

(ii) If the initial conditions are nonnegative and resetfree, $A_{(\cdot)}$ is a Metzler matrix; $d(\cdot) \geq 0$ and $c_{(\cdot)}$ and $b_{(.)}$have nonnegative components; then the output and the energy are positive for all time for all input with nonnegative components. This property as the previous one would still be kept under eventual positive additional control impulses [15] since the whole control action will kept its positive nature.

Note that positivity properties in the time domain are very relevant in the study of certain dynamic systems, like biological or epidemic ones, which, by nature, cannot have negative solutions at any time. See, for instance, [16-18]. The above properties follow from the positivity properties of the unforced and forced output solution trajectory in externally positive systems. However, those properties do not imply passivity without invoking additional conditions since the externally positive system can be nonstable, [19].

\section{Conflicts of Interest}

The author declares that he does not have any conflicts of interest.

\section{Acknowledgments}

This research is supported by the Spanish Government and by the European Fund of Regional Development FEDER through Grant DPI2015-64766-R and by UPV/EHU by Grant PGC 17/33.

\section{References}

[1] G. Zames, "On the input-output stability of time-varying nonlinear feedback systems part I: conditions derived using concepts of loop gain, conicity, and positivity," IEEE Transactions on Automatic Control, vol. 11, no. 2, pp. 228-238, 1966.

[2] N. Kottenstette and P. J. Antsaklis, "Relationships between positive real, passive dissipative, and positive systems," in Proceedings of the 2010 American Control Conference, ACC '10, pp. 409416, July 2010.

[3] B. A. Francis, A Course in $H_{\infty}$ Control Theory, Lecture Notes in Control and Information Sciences No. 88, Springer-Verlag, Heidelberg, Berlin, Germany, 1992.

[4] P. Dorato, L. Fortuna, and G. Muscato, Robust Control for Unstructured Perturbations- An Introduction, Lecture Notes in Control and Information Sciences No. 168, Springer-Verlag, Heidelberg, Berlin, Germany, 1992.

[5] B. Brogliato, R. Lozano, B. Maschke, and O. Egeland, "Dissipative systems analysis and control," in Theory and Applications, Springer-Verlag, Heidelberg, Berlin, Germany, 2nd edition, 2007.

[6] A. van der Schaft, L2-Gain and Passivity Techniques in Nonlinear Control, Springer-Verlag, Heidelberg, Berlin, Germany, 2nd edition, 2000.

[7] D. Hill and P. Moylan, "The stability of nonlinear dissipative systems," IEEE Transactions on Automatic Control, vol. 21, no. 5, pp. 708-711, 1976.

[8] M. Sagbas, K. Fidanboylu, and M. C. Bayram, "A new currentmode multifunction filter with high impedance outputs using minimum number of passive elements," in Proceedings of World Academy of Science Engineering and Technology, vol. 2, pp. 116119, 2005.

[9] M. de la Sen, "Stability of composite systems with an asymptotically hyperstable subsystem," International Journal of Control, vol. 44, no. 6, pp. 1769-1775, 1986.

[10] A. T. Ademola, S. Moyo, B. S. Ogundare, M. O. Ogundiran, and O. A. Adesina, "Stability and boundedness of solutions to a certain second-Order nonautonomous stochastic differential equation," International Journal of Analysis, vol. 2016, Article ID 2012315, 11 pages, 2016.

[11] C. Tunc and E. Tunc, "On the asymptotic behavior of solutions of certain second-order differential equations," Journal of The Franklin Institute, vol. 344, no. 5, pp. 391-398, 2007.

[12] C. Tunc and O. Tunc, "A note on certain qualitative properties of a second order linear differential system," Applied Mathematics and Information Sciences, vol. 9, no. 2, pp. 953-956, 2015.

[13] M. de la Sen, "Preserving positive realness through discretization," Positivity, vol. 6, no. 1, pp. 31-45, 2002.

[14] M. de la Sen and A. Ibeas, "On the global asymptotic stability of switched linear time-varying systems with constant point delays," Discrete Dynamics in Nature and Society, vol. 2005, no. 1, 67 pages, 2005.

[15] J. Tariboon, S. K. Ntouyas, and P. Agarwal, "New concepts of fractional quantum calculus and applications to impulsive fractional $q$ - difference equations," Advances in Difference Equations, vol. 2015, article 18, 2015.

[16] M. De la Sen, "The generalized Beverton-Holt equation and the control of populations," Applied Mathematical Modelling, vol. 32, no. 11, pp. 2312-2328, 2008.

[17] M. De La Sen and S. Alonso-Quesada, "Model-matching-based control of the beverton-holt equation in ecology," Discrete Dynamics in Nature and Society, vol. 2008, Article ID 793512, 21 pages, 2008.

[18] M. De la Sen and S. Alonso-Quesada, "Control issues for the Beverton-Holt equation in ecology by locally monitoring 
the environment carrying capacity: non-adaptive and adaptive cases," Applied Mathematics and Computation, vol. 215, no. 7, pp. 2616-2633, 2009.

[19] M. De la Sen, "On positivity of singular regular linear timedelay time-invariant systems subject to multiple internal and external incommensurate point delays," Applied Mathematics and Computation, vol. 190, no. 1, pp. 382-401, 2007. 


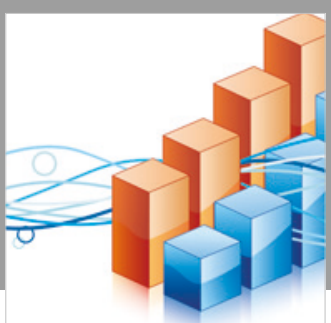

Advances in

Operations Research

\section{-n-m}
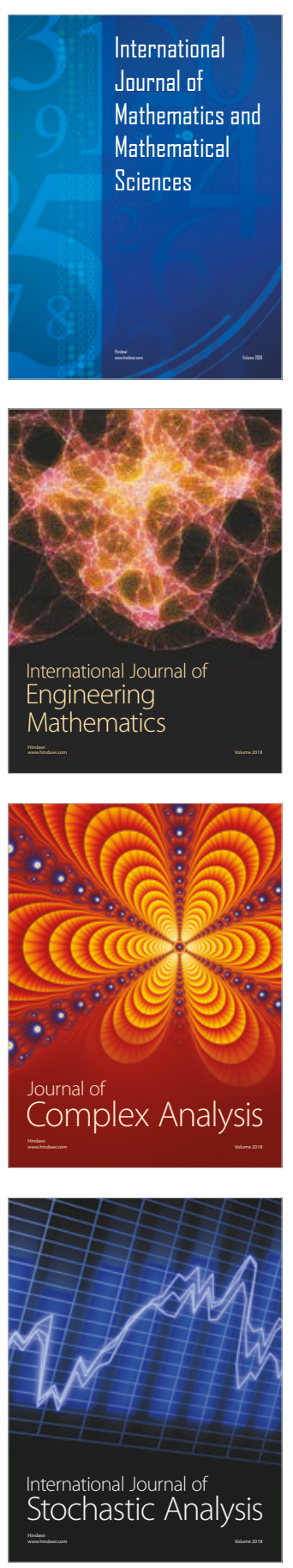
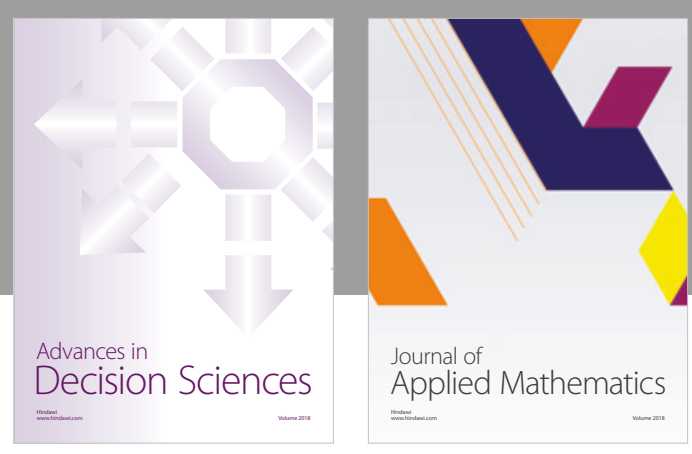

Journal of

Applied Mathematics
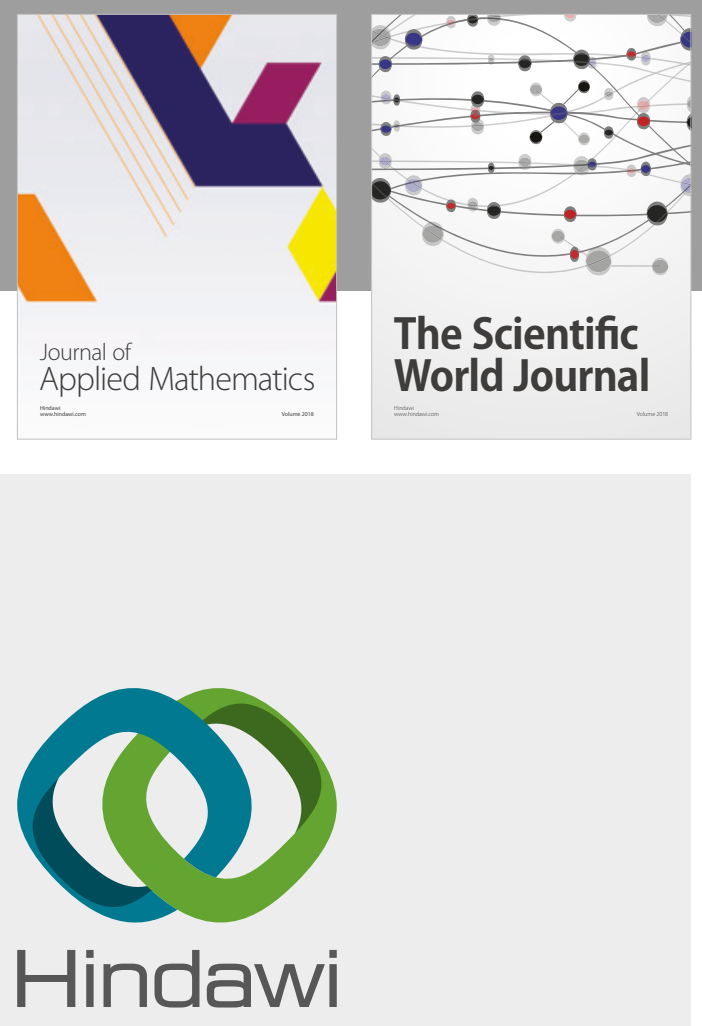

Submit your manuscripts at

www.hindawi.com

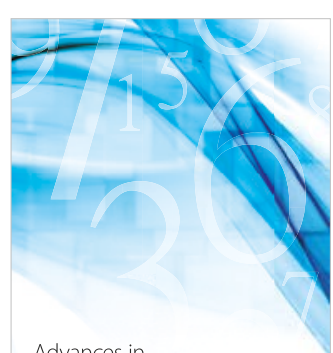

Advances in
Numerical Analysis
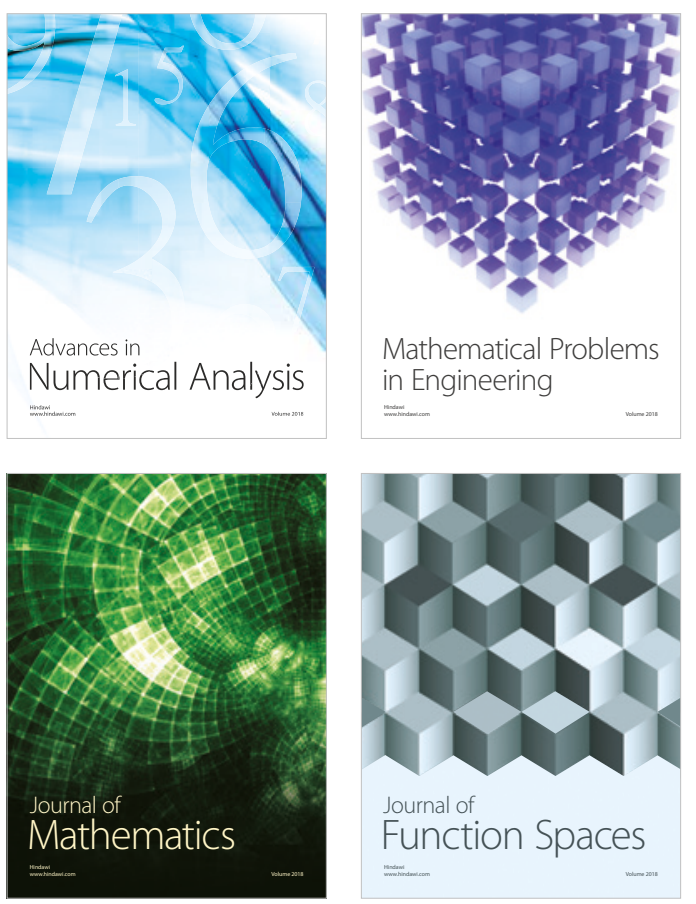

Mathematical Problems in Engineering

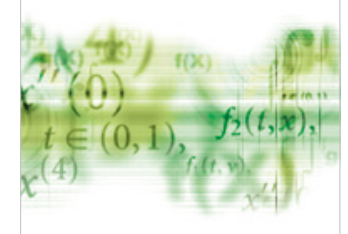

International Journal of

Differential Equations

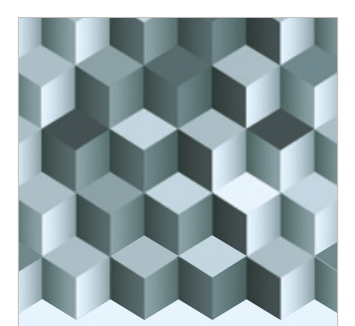

Journal of

Function Spaces

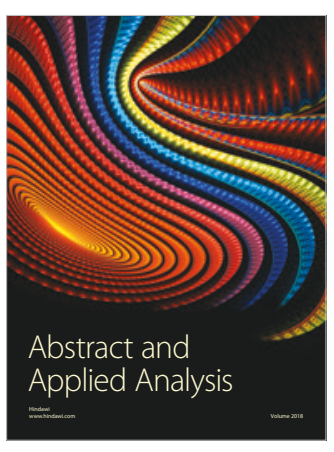

The Scientific

World Journal

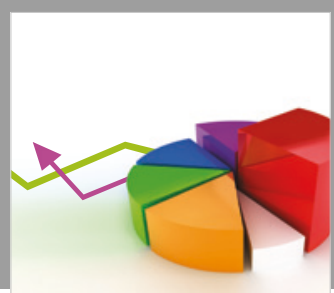

Journal of

Probability and Statistics
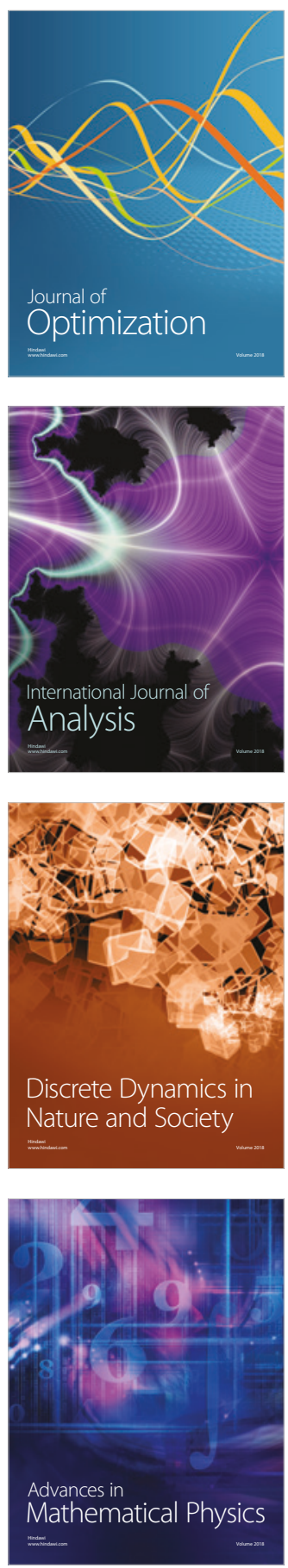